\title{
QUARTIC DEL PEZZO SURFACES OVER FUNCTION FIELDS OF CURVES
}

\author{
BRENDAN HASSETT AND YURI TSCHINKEL
}

\section{INTRODUCTION}

The geometry of spaces of rational curves of low degree on Fano threefolds is a very active area of algebraic geometry. One of the main goals is to understand the Abel-Jacobi morphism from the base of a family of such curves to the intermediate Jacobian of the threefold. For instance, does a given family of curves dominate the intermediate Jacobian? If so, what are the fibers of this morphism? Does it give the maximal rationally connected (MRC) quotient? Representative results in this direction are available for:

- cubic threefolds [HRS05, JHS, IM00, MT01;

- Fano threefolds of genus six and degree 10 in $\mathbb{P}^{7}$ DIM12;

- Fano threefolds of genus seven and degree 12 in $\mathbb{P}^{8}$ [IM07];

- moduli of vector bundles [Cas04] - this case makes clear that one cannot always expect the morphism to the intermediate Jacobian to give the MRC fibration.

At the same time, del Pezzo fibrations $\pi: \mathcal{X} \rightarrow \mathbb{P}^{1}$ are equally interesting geometrically. Moreover, the special case where the rational curves happen to be sections of $\pi$ is particularly important for arithmetic applications. It is a major open problem to determine whether or not sections exist over a non-closed ground field. Of course, the Tsen-Lang theorem gives sections when the ground field is algebraically closed. Even when there are rigid sections, these are typically defined over extensions of large degree.

However, suppose that the space of sections of fixed height is rationally connected over the intermediate Jacobian $\operatorname{IJ}(\mathcal{X})$. If the fibration is defined over a finite field and the space of sections descends to this field then $\pi$ has sections over that field. Indeed, this follows by combining a theorem of Lang [Lan55] (principal homogeneous spaces for abelian varities over finite fields are trivial) and a theorem of Esnault

Date: July 30, 2021. 
Esn03 (rationally connected varieties over finite fields have rational points). This point of view was developed for quadric surface bundles in [HT12b], with applications to effective versions of weak approximation.

This paper addresses the case of quartic del Pezzo fibrations $\pi$ : $\mathcal{X} \rightarrow \mathbb{P}^{1}$. Throughout, we assume $\mathcal{X}$ is smooth and the degenerate fibers of $\pi$ have at worst one ordinary singularity. We classify numerical invariants of the family - the fundamental invariant is the height, denoted $h(\mathcal{X})$. We provide explicit geometric realizations for fibrations of small height. We exhibit families of sections parametrizing the intermediate Jacobian and admitting rationally-connected fibrations over the intermediate Jacobian. The underlying constructions often involve Brill-Noether theory for smooth or nodal curves. Our main result is the comprehensive analysis of spaces of small-height sections for height 12 fibrations in Section [10, here $\mathcal{X}$ dominates a nodal Fano threefold of genus seven and degree 12.

Acknowledgments: The first author was supported by National Science Foundation Grants 0901645, 0968349, and 1148609; the second author was supported by National Science Foundation Grants 0739380, 0968318, and 1160859. We are grateful to Asher Auel, Jean-Louis Colliot-Thélène, and R. Parimala for useful conversations and Nikita Kozin for comments on the manuscript; we appreciate Jason Starr and Yi Zhu explaining the results of [Zhu11] on Abel-Jacobi morphisms for fibrations of rationally connected varieties over curves.

\section{Basic Properties of QUARTIC Del Pezzo Surfaces}

Let $X$ be a quartic del Pezzo surface over an algebraically closed field. $X$ is isomorphic to $\mathbb{P}^{2}$ blown up at five distinct points such that no three are collinear. Thus we may identify

$$
\operatorname{Pic}(X)=\mathbb{Z} L+\mathbb{Z} E_{1}+\mathbb{Z} E_{2}+\mathbb{Z} E_{3}+\mathbb{Z} E_{4}+\mathbb{Z} E_{5},
$$

where $L$ is the pull-back of the hyperplane class on $\mathbb{P}^{2}$ and the $E_{i}$ are the exceptional divisors. The primitive divisors

$$
\Lambda=K_{X}^{\perp} \subset \operatorname{Pic}(X)
$$

are a lattice under the intersection form; using the basis

$$
\left\{E_{1}-E_{2}, E_{2}-E_{3}, E_{3}-E_{4}, E_{4}-E_{5}, L-E_{1}-E_{2}-E_{3}\right\}
$$


we have

$$
\Lambda \simeq\left(\begin{array}{ccccc}
-2 & 1 & 0 & 0 & 0 \\
1 & -2 & 1 & 0 & 0 \\
0 & 1 & -2 & 1 & 1 \\
0 & 0 & 1 & -2 & 0 \\
0 & 0 & 1 & 0 & -2
\end{array}\right)
$$

Writing $\Lambda^{*}=\operatorname{Hom}(\Lambda, \mathbb{Z})$, the intersection form gives a surjection

$$
\operatorname{Pic}(X) \rightarrow \Lambda^{*}
$$

with kernel generated by $K_{X}$. Note that $\Lambda^{*} / \Lambda$ is a cyclic group of order four.

The action of the monodromy group on $\operatorname{Pic}(X)$ factors through the Weyl group $W\left(D_{5}\right)$ coming from the $D_{5}$ root system contained in the lattice $(-1) \Lambda$. Abstractly, $W\left(D_{5}\right)$ may be realized as an extension of the symmetric group

$$
1 \rightarrow(\mathbb{Z} / 2 \mathbb{Z})^{4} \rightarrow W\left(D_{5}\right) \rightarrow \mathfrak{S}_{5} \rightarrow 1
$$

concretely, this is the subgroup of the group of signed $5 \times 5$ permutation matrices where the determinant of the matrix equals the sign of the permutation.

The anticanonical model of $X$ is a complete intersection of two quadrics

$$
X=\left\{Q_{0}=Q_{1}=0\right\} \subset \mathbb{P}^{4} .
$$

Let $\operatorname{Gr}\left(2, \Gamma\left(\mathcal{O}_{\mathbb{P}^{4}}(2)\right)\right) \simeq \operatorname{Gr}(2,15)$ denote the Grassmannian parametrizing pencils of quadrics in $\mathbb{P}^{4}$. The group $\mathrm{SL}_{5}$ acts naturally on $\mathbb{P}^{4}$ and this Grassmannian, linearized via the Plücker embedding. Consider the loci of stable and semistable points

$$
U_{s} \subset U_{s s} \subset \operatorname{Gr}\left(2, \Gamma\left(\mathcal{O}_{\mathbb{P}^{4}}(2)\right)\right)
$$

and the resulting GIT quotient

$$
\bar{M}:=\operatorname{Gr}\left(2, \Gamma\left(\mathcal{O}_{\mathbb{P}^{4}}(2)\right)\right) / / \mathrm{SL}_{5} .
$$

Principal results of [MM93] include:

- $U_{s}$ coincides with the locus of smooth quartic del Pezzo surfaces;

- $U_{s s}$ parametrizes quartic del Pezzo surfaces with at worst ordinary double points;

- smooth quartic del Pezzo surfaces are diagonalizable, i.e., there exist coordinates $x_{0}, \ldots, x_{4}$ on $\mathbb{P}^{4}$ such that

$X=\left\{x_{0}^{2}+x_{1}^{2}+x_{2}^{2}+x_{3}^{2}+x_{4}^{2}=c_{0} x_{0}^{2}+c_{1} x_{1}^{2}+c_{2} x_{2}^{2}+c_{3} x_{3}^{2}+c_{4} x_{4}^{2}=0\right\} ;$ 
- closed orbits in $U_{s s}$ correspond to diagonalizable del Pezzo surfaces, which have 0,2 , or 4 ordinary double points;

- the determinantal scheme of a quartic del Pezzo surface

$$
X \mapsto Z=\left\{\operatorname{det}\left(s_{0} Q_{0}+s_{1} Q_{0}\right)=0\right\} \subset \mathbb{P}_{\left[s_{0}, s_{1}\right]}^{1}=\mathbb{P}\left(\Gamma\left(\mathcal{I}_{X}(2)\right)\right)
$$

induces a rational map

$$
\Xi: \operatorname{Gr}\left(2, \Gamma\left(\mathcal{O}_{\mathbb{P}^{4}}(2)\right)\right) \rightarrow \mathbb{P}\left(\Gamma\left(\mathcal{O}_{\mathbb{P}^{1}}(5)\right)\right) / / \mathrm{SL}_{2},
$$

regular on $U_{s s}$. Consequently, we obtain an isomorphism of projective schemes

$$
\bar{M} \simeq \mathbb{P}\left(\Gamma\left(\mathcal{O}_{\mathbb{P}^{1}}(5)\right)\right) / / \mathrm{SL}_{2} \simeq \bar{M}_{0,5} / \mathfrak{S}_{5},
$$

where $\bar{M}_{0,5}$ is the moduli space of stable rational curves with five marked points, with the canonical action of the symmetric group on five letters.

The diagonal representation of a smooth quartic del Pezzo surface has useful implications:

Consider the group $H=(\mathbb{Z} / 2 \mathbb{Z})^{4}$, realized as $5 \times 5$ diagonal matrices with \pm 1 on the diagonal modulo the subgroup $\pm I$. Then $H$ acts on $X$ and this action is transitive on the 16 lines of $X$.

The closure in $U_{s s}$ of the orbit of any quartic del Pezzo surface with a unique ordinary double point contains quartic del Pezzo surfaces with at least two ordinary double points. Indeed, quartic del Pezzo surfaces with one ordinary double point are strictly semistable but have orbits that are not closed. In particular, the moduli stack of quartic del Pezzo surfaces with ordinary singularities is non-separated. Concretely, fix a quartic del Pezzo surface $X=\left\{Q_{0}=Q_{1}=0\right\}$ with one ordinary singularity. Supposing the singularity is at $[0,0,0,0,1]$ with tangent space $x_{0}=0$, and also that $Q_{0}$ cuts out this tangent space and $Q_{1}$ is nodal at $[0,0,0,0,1]$, we obtain

$$
Q_{0}=x_{0} x_{4}+Q_{2}\left(x_{0}, x_{1}, x_{2}, x_{3}\right), \quad Q_{1}=Q_{1}\left(x_{0}, x_{1}, x_{2}, x_{3}\right) .
$$

Writing $Q_{2}=x_{0} \ell_{2}\left(x_{0}, x_{1}, x_{2}, x_{3}\right)+R_{2}\left(x_{1}, x_{2}, x_{3}\right)$ and substituting $x_{4}=$ $x_{4}^{\prime}-\ell_{2}$, we obtain

$$
X=\left\{x_{4}^{\prime} x_{0}+R_{2}\left(x_{1}, x_{2}, x_{3}\right)=Q_{1}=0\right\} .
$$

Writing $Q_{1}=x_{0} \ell_{1}\left(x_{0}, x_{1}, x_{2}, x_{3}\right)+R_{1}\left(x_{1}, x_{2}, x_{3}\right)$ yields

$$
X=\left\{x_{4}^{\prime} x_{0}+R_{2}\left(x_{1}, x_{2}, x_{3}\right)=x_{0} \ell_{1}+R_{1}\left(x_{1}, x_{2}, x_{3}\right)=0\right\} .
$$


QUARTIC DEL PEZZO SURFACES OVER FUNCTION FIELDS OF CURVES 5

Consider the action of the one-parameter subgroup $\rho(t)$ given by

$$
x_{0} \mapsto t x_{0}, x_{i} \mapsto x_{i}, i=1,2,3, x_{4}^{\prime} \mapsto t^{-1} x_{4}^{\prime} .
$$

The limit

$$
X_{0}:=\lim _{t \rightarrow 0} \rho(t) \cdot X=\left\{x_{4}^{\prime} x_{0}+R_{2}\left(x_{1}, x_{2}, x_{3}\right)=R_{1}\left(x_{1}, x_{2}, x_{3}\right)=0\right\}
$$

has double points $[0,0,0,0,1]$ and $[1,0,0,0,0]$. Moreover, when $\left\{R_{1}=\right.$ $\left.R_{2}=0\right\} \subset \mathbb{P}^{2}$ consists of four distinct points we have

$$
\operatorname{Aut}^{\circ}\left(X_{0}\right) \simeq \mathbb{G}_{m}
$$

i.e., the identity component of the automorphism group corresponds to $\rho(t)$.

It is a fundamental result of Geometric Invariant Theory that the failure of separatedness is governed by specializations under one-parameter subgroups arising as automorphisms of objects with closed orbit (see $[\mathrm{HH}$, Prop. 4.2]). Note that there is a unique non-closed orbit in the basin of attraction of $X_{0}$ under $\rho(t)$. Thus removing the semistable objects of type $X_{0}$ eliminates the non-separatedness. Alternately, we could apply the Kirwan blow-up process [Kir85] to eliminate semistable points with positive-dimensional stabilizers.

We summarize this discussion as follows:

Proposition 1. Let $\mathcal{M}$ denote the moduli stack of quartic del Pezzo surfaces with at worst one ordinary double point, with coarse moduli space $M$. Then we have

- $\Xi$ (defined in (2.2)) induces a morphism of stacks

$$
\xi: \mathcal{M} \rightarrow\left[\left(\overline{\mathcal{M}}_{0,5} \backslash S\right) / \mathfrak{S}_{5}\right]
$$

where $S$ is the zero-dimensional boundary stratum (see Figure 11);

- $\xi$ induces an isomorphism of coarse moduli spaces

$$
M \stackrel{\sim}{\rightarrow}\left(\bar{M}_{0,5} \backslash S\right) / \mathfrak{S}_{5}
$$

The invariants of binary quintics were computed in the 19th century; see [Bak08] for a description convenient for our purposes. It follows that

$$
M \simeq \mathbb{P}(1,2,3) \backslash s
$$

where $s$ is the image of $S$, and a point where the minimal degree invariant is non-zero. Thus we obtain

Corollary 2. $\operatorname{Pic}(M) \simeq \mathbb{Z}$. 


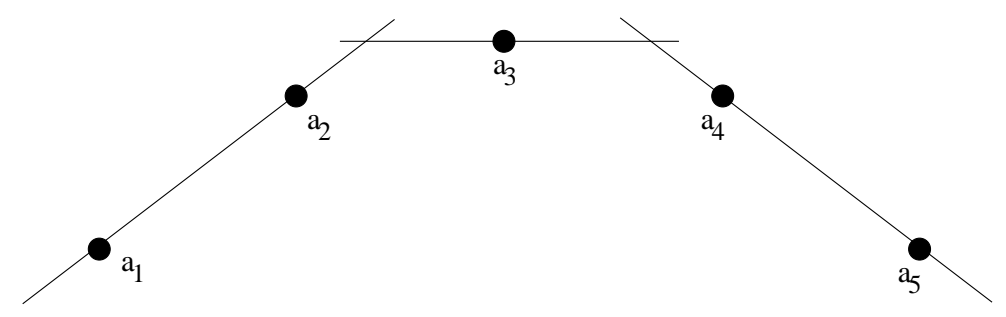

FiguRE 1. The zero-dimensional boundary stratum

\section{Quartic Del Pezzo fiber spaces over $\mathbb{P}^{1}$}

We work over an algebraically closed field of characteristic different from two.

Proposition 3. Let $\mathcal{X}$ be a smooth projective threefold and $\pi: \mathcal{X} \rightarrow \mathbb{P}^{1}$ a morphism such that the generic fiber is a smooth rational surface. Then

- $\mathcal{X}$ is separably rationally connected;

- the Hodge numbers $h^{i j}(\mathcal{X})=0$ for $(i, j) \neq(0,0),(1,1),(2,2)$, $(3,3),(1,2),(2,1)$.

Proof. $\pi$ admits a section $s: \mathbb{P}^{1} \rightarrow \mathcal{X}$. This follows from general results dJS03 or by the classification of rational surfaces and the TsenLang theorem (cf. [Has09]). Since the smooth fibers of $\pi$ are rational surfaces, there exist very free curves in the fibers incident to $s\left(\mathbb{P}^{1}\right)$. The techniques of GHS03 thus yield a very free section of $\pi$, proving separably rational connectedness.

It follows [Kol96, IV.3.8] that

$$
\Gamma\left(\Omega_{\mathcal{X}}^{1}\right)=\Gamma\left(\Omega_{\mathcal{X}}^{2}\right)=\Gamma\left(\Omega_{\mathcal{X}}^{3}\right)=0
$$

and by Serre duality

$$
H^{3}\left(\Omega_{\mathcal{X}}^{2}\right)=H^{3}\left(\Omega_{\mathcal{X}}^{1}\right)=H^{3}\left(\mathcal{O}_{\mathcal{X}}\right)=0 .
$$

The Leray spectral sequence for $\pi$ and Serre duality also give

$$
H^{1}\left(\mathcal{O}_{\mathcal{X}}\right)=H^{2}\left(\mathcal{O}_{\mathcal{X}}\right)=0 \quad H^{2}\left(\Omega_{\mathcal{X}}^{3}\right)=H^{1}\left(\Omega_{\mathcal{X}}^{3}\right)=0 .
$$

Assume that $\pi: \mathcal{X} \rightarrow \mathbb{P}^{1}$ is a fibration of quartic del Pezzo surfaces, with $\mathcal{X}$ smooth. The fibration has square-free discriminant if the fibers are complete intersections of two quadrics with at worst one ordinary 
double point; we assume this from now on. Under these assumptions, the relative dualizing sheaf $\omega_{\pi}$ is invertible, the direct image of its dual

$$
\pi_{*} \omega_{\pi}^{-1}
$$

is locally free, and the higher direct images vanish. Cohomology-andbase-change and the fact that $\omega_{\pi}^{-1}$ is very ample on fibers of $\pi$ yield an embedding $\mathcal{X} \subset \mathbb{P}\left(\left(\pi_{*} \omega_{\pi}^{-1}\right)^{\vee}\right)$. Let $\mathcal{I}_{\mathcal{X}}(2)$ denote the ideal sheaf of $\mathcal{X}$ twisted by $\mathcal{O}_{\mathbb{P}\left(\left(\pi_{*} \omega_{\pi}^{-1}\right)^{\vee}\right)}(2)$; then $\pi_{*}\left(\mathcal{I}_{\mathcal{X}}(2)\right) \subset \operatorname{Sym}^{2}\left(\pi_{*} \omega_{\pi}^{-1}\right)$ is a subbundle of rank two with quotient $\pi_{*} \omega_{\pi}^{-2}$.

Proposition 4. Let $\pi: \mathcal{X} \rightarrow \mathbb{P}^{1}$ be a fibration of quartic del Pezzo surfaces with square-free discriminant, defined over a field of characteristic $p>2$. Then $\mathcal{X}$ and $\pi$ are liftable to characteristic zero.

Remark 5. Assuming Proposition 4, the Hodge-de Rham spectral sequence for $\mathcal{X}$ degenerates provided $p>3($ or $=0)$ [DI87]. Thus $h^{11}(\mathcal{X})$ equals the Picard rank of $\mathcal{X}$, and $h^{11}$ and $h^{12}$ are unchanged on reduction to $\mathcal{X}$ from characteristic zero.

Proof. Every vector bundle over $\mathbb{P}^{1}$ decomposes as a direct sum of line bundles

$$
V \simeq \oplus_{i=1}^{r} \mathcal{O}_{\mathbb{P}^{1}}\left(a_{i}\right)
$$

Clearly any such direct sum lifts to characteristic zero, i.e., lift each summand individually. Cohomology commutes with reduction modulo $p$ for such a lift; given lifts of two such bundles, taking homomorphisms between them commutes with reduction as well.

In particular, we may lift the decomposition of $V=\pi_{*} \omega_{\pi}^{-1}$ to characteristic zero; the same holds true for $\operatorname{Sym}^{2} V$ and its quotient $V^{\prime}=$ $\pi_{*} \omega_{\pi}^{-2}$. There is a combinatorial criterion [Sha78] for which vector bundles arise as subbundles of a given bundle, independent of the characteristic. In particular, the quotient homomorphism

$$
\mathrm{Sym}^{2} V \rightarrow V^{\prime}
$$

lifts to characteristic zero, as does its kernel. The resulting rank-two subbundle gives the defining equations of the lift of $\mathcal{X}$ to characteristic zero. Having square-free discriminant is an open condition, so the resulting lift shares this property.

We define

$$
h(\mathcal{X}):=\operatorname{deg}\left(c_{1}\left(\omega_{\pi}\right)^{3}\right)
$$

to be the height of the del Pezzo surface fibration. Corollary 2 implies this is the unique numerical invariant for families with square-free discriminant: 
Proposition 6. Let $\pi: \mathcal{X} \rightarrow \mathbb{P}^{1}$ be a quartic del Pezzo fibration with square-free discriminant. Then we have:

- $h(\mathcal{X})=-2 \operatorname{deg}\left(\pi_{*} \omega_{\pi}^{-1}\right)$;

- $\Delta:=$ number of singular fibers of $\pi=2 h(\mathcal{X})$;

- the topological Euler characteristic

$$
\begin{gathered}
\begin{aligned}
\chi(\mathcal{X}) & =(2-\Delta) \cdot \chi(\text { smooth fiber })+\Delta \cdot \chi(\text { singular fiber }) \\
& =16-2 h(\mathcal{X}) ;
\end{aligned} \\
\text { - } \chi\left(\Omega_{\mathcal{X}}^{1}\right)=1-\chi(\mathcal{X}) / 2=h(\mathcal{X})-7 ; \\
\text { - expected number of parameters }=-\chi\left(T_{\mathcal{X}}\right)=\frac{3}{2} h(\mathcal{X})-1 .
\end{gathered}
$$

Proof. The quantities $h(\mathcal{X}), \operatorname{deg}\left(\pi_{*} \omega_{\pi}^{-1}\right)$, and $\Delta$ all depend on the degree of the classifying map $\mu: \mathbb{P}^{1} \rightarrow \mathcal{M}$, thus are in constant proportion. To evaluate the proportionality constant, it suffices to consider a singular non-constant example, e.g., the intersection of a quadric in $\mathbb{P}^{4}$ with a pencil of quadric hypersurfaces. (See Case 1 of Section 4 for details.)

Once we know the number of singular fibers, it is straightforward to compute the (étale) topological Euler characteristic. The Euler characteristics of $\Omega_{\mathcal{X}}^{1}$ and $\Omega_{\mathcal{X}}^{2}$ are related to the topological Euler characteristic via the Hodge-de Rham spectral sequence

$$
\chi(\mathcal{X})=\chi\left(\mathcal{O}_{X}\right)-\chi\left(\Omega_{\mathcal{X}}^{1}\right)+\chi\left(\Omega_{\mathcal{X}}^{2}\right)-\chi\left(\Omega_{\mathcal{X}}^{3}\right)
$$

Serre duality and Proposition 3 give

$$
\chi(\mathcal{X})=2-2 \chi\left(\Omega_{\mathcal{X}}^{1}\right)
$$

Finally, the expected number of parameters $-\chi\left(T_{\mathcal{X}}\right)$, which may be computed by Hirzebruch-Riemann-Roch and Gauss-Bonnet:

$$
\begin{aligned}
\chi\left(T_{\mathcal{X}}\right) & =\frac{1}{2} c_{1}\left(T_{\mathcal{X}}\right)^{3}-\frac{19}{24} c_{1}\left(T_{\mathcal{X}}\right) c_{2}\left(T_{\mathcal{X}}\right)+\frac{1}{2} c_{3}\left(T_{\mathcal{X}}\right) \\
& =-\frac{1}{2} c_{1}\left(\omega_{\mathcal{X}}\right)^{3}-19 \chi\left(\mathcal{O}_{\mathcal{X}}\right)+\frac{1}{2} \chi(\mathcal{X}) .
\end{aligned}
$$

We have

$$
c_{1}\left(\omega_{\mathcal{X}}\right)^{3}=\left(c_{1}\left(\pi^{*} \omega_{\mathbb{P}^{1}}\right)+c_{1}\left(\omega_{\pi}\right)\right)^{3}=h(\mathcal{X})-24
$$

whence

$$
\chi\left(T_{\mathcal{X}}\right)=-\frac{1}{2} h(\mathcal{X})+12-19+8-h(\mathcal{X})=-\frac{3}{2} h(\mathcal{X})+1,
$$

as claimed.

Combining with Proposition 3 and Remark 5, we obtain 
Corollary 7. Retain the notation and assumptions of Proposition [; assume the characteristic $p>3$ (or $=0$ ) and set

$$
h^{11}=\operatorname{dim} H^{1}\left(\Omega_{\mathcal{X}}^{1}\right)=\operatorname{rank}(\operatorname{Pic}(\mathcal{X})) .
$$

Then the Hodge diamond of $\mathcal{X}$ takes the form

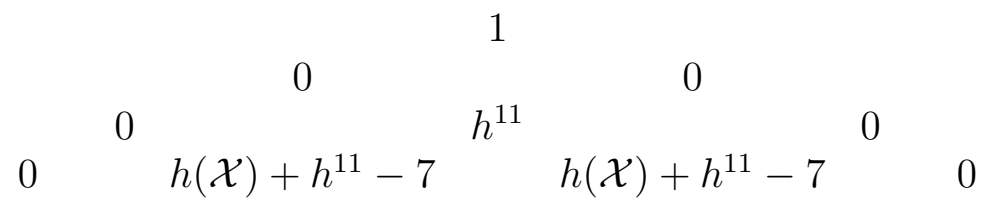

This can be interpreted in geometric terms: Let $\pi: \mathcal{X} \rightarrow \mathbb{P}^{1}$ be a fibration of quartic del Pezzo surfaces with square-free discriminant. Let $D \rightarrow \mathbb{P}^{1}$ be the degree-five covering parametrizing nodal quadric hypersurfaces containing the fibers of $\pi$, and $\tilde{D} \rightarrow D$ the double covering parametrizing rulings of these quadric hypersurfaces. The assumption on singular fibers implies that $D \rightarrow \mathbb{P}^{1}$ is simply branched and $\tilde{D} \rightarrow D$ is étale, and we may consider the resulting Prym variety $\operatorname{Prym}(\tilde{D} \rightarrow D)$.

Proposition 8. Retaining the notation above, let $A^{2}(\mathcal{X})$ denote codimension two cycles algebraically equivalent to zero. Then $A^{2}(\mathcal{X}) \simeq$ $\operatorname{Prym}(\tilde{D} \rightarrow D)$.

Proof. Fix a section $s: \mathbb{P}^{1} \rightarrow \mathcal{X}$ of $\pi$, not passing through any lines of the singular fibers. Projecting from $s$ realizes the generic fiber of $\pi$ as a conic bundle over $\mathbb{P}^{1}$. Indeed, blowing up a quartic del Pezzo surface at a point not on a line yields a cubic surface with a line; projecting the cubic surface from that line gives a conic bundle over $\mathbb{P}^{1}$. There are five degenerate fibers, corresponding to the generic fiber of $D \rightarrow \mathbb{P}^{1}$, and ten irreducible components in these fibers, corresponding to the the generic fiber of $\tilde{D} \rightarrow \mathbb{P}^{1}$. Thus the blow-up of $\mathcal{X}$ along $s\left(\mathbb{P}^{1}\right)$ is a conic bundle over a Hirzebruch surface surface, with discriminant curve $D$ and étale cover $\tilde{D} \rightarrow D$. A straightforward variation on the argument of $[$ Bea77, $\S 3]$ implies that $A^{2}\left(\operatorname{Bl}_{s\left(\mathbb{P}^{1}\right)}(\mathcal{X})\right) \simeq \operatorname{Prym}(\tilde{D} \rightarrow D)$; the same holds for $A^{2}(\mathcal{X})$. (For related results, see [Kan89].)

Remark 9. Over $\mathbb{C}$, this Prym variety is the intermediate Jacobian $\mathrm{IJ}(\mathcal{X})$; its construction is compatible with reduction modulo $p$, assuming the reduction still has square-free discriminant. Even over fields of characteristic $p>0, \operatorname{Prym}(\tilde{D} \rightarrow D)$ has the requisite universal 
property for one-cycles [Bea77, §3.2]: Given a family of curves

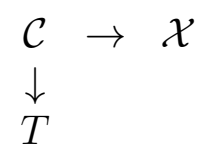

over a smooth connected base $T$, there is an Abel-Jacobi morphism $T \rightarrow \operatorname{Prym}(\tilde{D} \rightarrow D)$.

The Lefschetz hyperplane theorem gives:

Proposition 10. Let $\mathcal{X} \rightarrow \mathbb{P}^{1}$ be a fibration of quartic del Pezzo surfaces. Suppose $\mathcal{X} \subset \mathbb{P}\left(\left(\pi_{*} \omega_{\pi}^{-1}\right)^{\vee}\right)$ is a smooth complete intersection of ample divisors in $\mathbb{P}\left(\left(\pi_{*} \omega_{\pi}^{-1}\right)^{\vee}\right)$. Then $\operatorname{Pic}(\mathcal{X})=\mathbb{Z}^{2}$.

Definition 11. Let $\pi: \mathcal{X} \rightarrow \mathbb{P}^{1}$ be a quartic del Pezzo fibration with square-free discriminant. The anticanonical height of a section $\sigma: \mathbb{P}^{1} \rightarrow \mathcal{X}$ is defined

$$
h_{\omega_{\pi}^{-1}}(\sigma)=\operatorname{deg}\left(\sigma^{*} \omega_{\pi}^{-1}\right) .
$$

Note that this equals the degree of the normal bundle $N_{\sigma}$, thus the Hilbert scheme of sections has dimension $\geq h_{\omega_{\pi}^{-1}}(\sigma)+2$ at $\sigma$.

\section{EXPLICIT REALIZATION OF GENERIC FAMILIES}

In this section, we give concrete geometric realizations of families of each height. We start by writing

$$
\begin{gathered}
\pi_{*} \omega_{\pi}^{-1}=\mathcal{O}_{\mathbb{P}^{1}}\left(-a_{1}\right) \oplus \mathcal{O}_{\mathbb{P}^{1}}\left(-a_{2}\right) \oplus \mathcal{O}_{\mathbb{P}^{1}}\left(-a_{3}\right) \oplus \mathcal{O}_{\mathbb{P}^{1}}\left(-a_{4}\right) \oplus \mathcal{O}_{\mathbb{P}^{1}}\left(-a_{5}\right), \\
a_{1} \leq a_{2} \leq a_{3} \leq a_{4} \leq a_{5} .
\end{gathered}
$$

We refer the reader to [Shr06, 2.2] for a discussion of which $a_{j}$ may occur. Here we assume this sheaf is generic, in the sense that it is as 'balanced' as possible, i.e., $a_{5}-a_{1} \leq 1$. Thus $\pi_{*} \omega_{\pi}^{-1}$ is one of the following:

(1) $\mathcal{O}_{\mathbb{P}^{1}}^{5} \otimes \mathcal{O}_{\mathbb{P}^{1}}(-m)$

(2) $\left(\mathcal{O}_{\mathbb{P}^{1}} \oplus \mathcal{O}_{\mathbb{P}^{1}}(-1)^{4}\right) \otimes \mathcal{O}_{\mathbb{P}^{1}}(-m)$

(3) $\left(\mathcal{O}_{\mathbb{P}^{1}}^{2} \oplus \mathcal{O}_{\mathbb{P}^{1}}(-1)^{3}\right) \otimes \mathcal{O}_{\mathbb{P}^{1}}(-m)$

(4) $\left(\mathcal{O}_{\mathbb{P}^{1}}^{3} \oplus \mathcal{O}_{\mathbb{P}^{1}}(-1)^{2}\right) \otimes \mathcal{O}_{\mathbb{P}^{1}}(-m)$

(5) $\left(\mathcal{O}_{\mathbb{P}^{1}}^{4} \oplus \mathcal{O}_{\mathbb{P}^{1}}(-1)\right) \otimes \mathcal{O}_{\mathbb{P}^{1}}(-m)$

where $m \in \mathbb{Z}$. In this situation, Proposition 6 may be proven by direct computations with resolutions. 
QUARTIC DEL PEZZO SURFACES OVER FUNCTION FIELDS OF CURVES 11

Case 1. We compute invariants for generic quartic del Pezzo surfaces

$$
\pi: \mathcal{X} \rightarrow \mathbb{P}^{1}
$$

arising from morphisms into the Hilbert scheme

$$
\mu: \mathbb{P}^{1} \rightarrow \operatorname{Gr}\left(2, \Gamma\left(\mathcal{O}_{\mathbb{P}^{4}}(2)\right)\right)
$$

of degree $d \geq 0$, where $\mu$ is an embedding transverse to the discriminant. In this situation, we may embed

$$
\mathcal{X} \subset P:=\mathbb{P}^{1} \times \mathbb{P}^{4},
$$

where

$$
\pi_{*} I_{\mathcal{X}}(2) \subset \Gamma\left(\mathcal{O}_{\mathbb{P}^{4}}(2)\right) \otimes \mathcal{O}_{\mathbb{P}^{1}}
$$

is a rank-two vector subbundle of degree $-d$.

Even part. Assuming this bundle is generic and $d=2 n$, we have

$$
\pi_{*} I_{\mathcal{X}}(2) \simeq \mathcal{O}_{\mathbb{P}^{1}}(-n)^{2}
$$

and $\mathcal{X}$ is a complete intersection of two forms of bidegree $(n, 2)$. As such, $\mathcal{X}$ depends on

$$
2(15(n+1)-2)-(3+24)=30 n-1
$$

parameters. In this situation

$$
\omega_{\pi}^{-1}=\mathcal{O}_{\mathcal{X}}(-2 n, 1), \quad \pi_{*} \omega_{\pi}^{-1}=\mathcal{O}_{\mathbb{P}^{1}}(-2 n) \otimes \Gamma\left(\mathcal{O}_{\mathbb{P}^{4}}(1)\right)
$$

whence

$$
\operatorname{deg}\left(\pi_{*} \omega_{\pi}^{-1}\right)=-10 n, \quad h(\mathcal{X})=20 n
$$

We compute the middle cohomology using the exact sequence

$$
0 \rightarrow I_{\mathcal{X}} / I_{\mathcal{X}}^{2} \rightarrow \Omega_{P}^{1} \mid \mathcal{X} \rightarrow \Omega_{\mathcal{X}}^{1} \rightarrow 0
$$

where the conormal bundle

$$
I_{\mathcal{X}} / I_{\mathcal{X}}^{2}=\mathcal{O}_{\mathcal{X}}(-n,-2)^{2} .
$$

Thus we find

$$
\chi\left(\Omega_{\mathcal{X}}^{1}\right)=\chi\left(\Omega_{P}^{1} \mid \mathcal{X}\right)-2 \chi\left(\mathcal{O}_{\mathcal{X}}(-n,-2)\right) .
$$

Using the Koszul resolution

$$
0 \rightarrow \mathcal{O}_{P}(-2 n,-4) \rightarrow \mathcal{O}_{P}(-n,-2)^{2} \rightarrow I_{\mathcal{X}} \rightarrow 0
$$

we compute

$$
\begin{aligned}
\chi\left(\mathcal{O}_{\mathcal{X}}(a, b)\right) & =\chi\left(\mathcal{O}_{P}(a, b)\right)-2 \chi\left(\mathcal{O}_{P}(a-n, b-2)\right)+\chi\left(\mathcal{O}_{P}(a-2 n, b-4)\right) \\
\chi\left(\Omega_{P}^{1} \mid \mathcal{X}(a, b)\right) & =\chi\left(\Omega_{P}^{1}(a, b)\right)-2 \chi\left(\Omega_{P}^{1}(a-n, b-2)\right)+\chi\left(\Omega_{P}^{1}(a-2 n, b-4)\right) .
\end{aligned}
$$


The formulas

$$
\begin{gathered}
\chi\left(\mathcal{O}_{P}(a, b)\right)=(a+1)\left(\begin{array}{c}
b+4 \\
4
\end{array}\right) \\
\chi\left(\Omega_{P}^{1}(a, b)\right)=(a-1)\left(\begin{array}{c}
b+4 \\
4
\end{array}\right)+\left(5\left(\begin{array}{c}
b+3 \\
4
\end{array}\right)-\left(\begin{array}{c}
b+4 \\
4
\end{array}\right)\right)(a+1),
\end{gathered}
$$

thus give

$$
\chi\left(\Omega_{\mathcal{X}}^{1}\right)=-7+20 n, \quad \chi(\mathcal{X})=16-40 n .
$$

When $n>0$ we obtain

$$
h^{2}\left(\Omega_{\mathcal{X}}^{1}\right)=-5+20 n
$$

as well.

Remark 12. The special case $n=0$ is a constant family of quartic del Pezzo surfaces, which clearly has sections.

Odd part. Now assume $d=2 n+1$ so (4.1) takes the form

$$
\pi_{*} I_{\mathcal{X}}(2) \simeq \mathcal{O}_{\mathbb{P}^{1}}(-n) \oplus \mathcal{O}_{\mathbb{P}^{1}}(-n-1)
$$

and $\mathcal{X}$ is a complete intersection of forms of bidegrees $(n, 2)$ and $(n+$ $1,2)$. In this situation

$$
\omega_{\pi}^{-1}=\mathcal{O}_{\mathcal{X}}(-2 n-1,1), \quad \pi_{*} \omega_{\pi}^{-1}=\mathcal{O}_{\mathbb{P}^{1}}(-2 n-1) \otimes \Gamma\left(\mathcal{O}_{\mathbb{P}^{4}}(1)\right)
$$

whence

$$
\operatorname{deg}\left(\pi_{*} \omega_{\pi}^{-1}\right)=-10 n-5, \quad h(\mathcal{X})=20 n+10 .
$$

Remark 13. The base case $n=0$ is studied in Section 9, where we show that it always admits a section $\sigma: \mathbb{P}^{1} \rightarrow \mathcal{X}$, defined over the coefficient field.

Case 2. We next consider

$$
\mathcal{X} \subset P:=\mathbb{P}^{1} \times \mathbb{P}^{5}
$$

contained in a complete intersection of a form $L$ of bidegree $(1,1)$.

Even part. Consider when $\mathcal{X}$ is a complete intersection of a form $L$ of bidegree $(1,1)$ and two forms of bidegree $(n, 2)$. Let $V$ be defined by the exact sequence

$$
0 \rightarrow \mathcal{O}_{\mathbb{P}^{1}}(-1) \stackrel{\cdot L}{\rightarrow} \Gamma\left(\mathcal{O}_{\mathbb{P}^{5}}(1)\right) \otimes \mathcal{O}_{\mathbb{P}^{1}} \rightarrow V \rightarrow 0 .
$$

For generic $L$, we have

$$
V \simeq \mathcal{O}_{\mathbb{P}^{1}}^{4} \oplus \mathcal{O}_{\mathbb{P}^{1}}(1)
$$

We have

$$
\omega_{\pi}^{-1}=\mathcal{O}_{\mathcal{X}}(-2 n-1,1)
$$


QUARTIC DEL PEZZO SURFACES OVER FUNCTION FIELDS OF CURVES 13 and the projection formula yields

$$
\pi_{*} \omega_{\pi}^{-1}=\mathcal{O}_{\mathbb{P}^{1}}(-2 n-1) \otimes V
$$

whence

$$
\operatorname{deg}\left(\pi_{*} \omega_{\pi}^{-1}\right)=4(-2 n-1)-2 n=-10 n-4, \quad h(\mathcal{X})=20 n+8 .
$$

Remark 14. We analyze rational points in the case $n=0$. Consider the projection $\mathcal{Y}:=\pi_{2}(\mathcal{X}) \subset \mathbb{P}^{5}$ onto the second factor; it is a complete intersection of two quadrics. The fibration corresponds to a pencil of hyperplane sections of $\mathcal{Y}$. Let $Z \subset \mathcal{Y}$ be its base locus, so that $\mathcal{X}=\mathrm{Bl}_{Z}(\mathcal{Y})$. Since $\mathcal{X}$ is smooth, $Z$ must be a smooth curve of genus one.

The constant subfamily

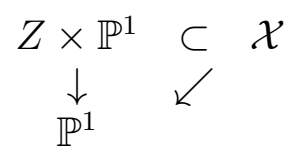

has a section over the coefficient field $k$, provided $Z(k) \neq \emptyset$.

Odd part. Now consider

$$
\mathcal{X} \subset P:=\mathbb{P}^{1} \times \mathbb{P}^{5}
$$

as a complete intersection of a form $L$ of bidegree $(1,1)$, a form of bidegree $(n, 2)$, and a form of bidegree $(n+1,2)$. We have

$$
\omega_{\pi}^{-1}=\mathcal{O}_{\mathcal{X}}(-2 n-2,1)
$$

so that

$$
\pi_{*} \omega_{\pi}^{-1}=\mathcal{O}_{\mathbb{P}^{1}}(-2 n-2) \otimes V,
$$

where $V$ is as above. Thus we conclude

$\operatorname{deg}\left(\pi_{*} \omega_{\pi}^{-1}\right)=4(-2 n-2)+(-2 n-1)=-10 n-9, \quad h(\mathcal{X})=20 n+18$.

Case 3. Consider

$$
\mathcal{X} \subset \mathbb{P}^{1} \times \mathbb{P}^{6}
$$

as a complete intersection of two forms $L_{1}$ and $L_{2}$ of bidegree $(1,1)$ and two additional forms quadratic in the second factor. Let $V$ be defined by the exact sequence

$$
0 \rightarrow \mathcal{O}_{\mathbb{P}^{1}}(-1)^{2} \stackrel{\cdot\left(L_{1}, L_{2}\right)}{\rightarrow} \Gamma\left(\mathcal{O}_{\mathbb{P}^{6}}(1)\right) \otimes \mathcal{O}_{\mathbb{P}^{1}} \rightarrow V \rightarrow 0 ;
$$

for generic $L_{1}$ and $L_{2}$, we have

$$
V \simeq \mathcal{O}_{\mathbb{P}^{1}}(1)^{2} \oplus \mathcal{O}_{\mathbb{P}^{1}}^{3}
$$


As before, we have

$$
\pi_{*} \mathcal{O}_{\mathcal{X}}(1) \simeq V
$$

Even part. Suppose that the additional quadratic forms both have degree $(n, 2)$. Then $\omega_{\pi} \simeq \mathcal{O}_{\mathcal{X}}(2 n+2,-1)$ whence

$$
\pi_{*} \omega_{\pi}^{-1} \simeq \mathcal{O}_{\mathbb{P}^{1}}(-2 n-1)^{2} \oplus \mathcal{O}_{\mathbb{P}^{1}}(-2 n-2)^{3}
$$

and

$$
\operatorname{deg}\left(\pi_{*} \omega_{\pi}^{-1}\right)=-10 n-8, \quad h(\mathcal{X})=20 n+16
$$

Odd part. Suppose that the additional forms have degrees $(n, 2)$ and $(n+1,2)$. Then $\omega_{\pi} \simeq \mathcal{O}_{\mathcal{X}}(2 n+3,-1)$ whence

$$
\pi_{*} \omega_{\pi}^{-1} \simeq \mathcal{O}_{\mathbb{P}^{1}}(-2 n-2)^{2} \oplus \mathcal{O}_{\mathbb{P}^{1}}(-2 n-3)^{3}
$$

and

$$
\operatorname{deg}\left(\pi_{*} \omega_{\pi}^{-1}\right)=-10 n-13, \quad h(\mathcal{X})=20 n+26 .
$$

Example 15 ( $n=-1$ case). We do the $n=-1$ case via a slightly different construction: Consider the intersection of the forms $L_{1}$ and $L_{2}$ :

$$
\mathbb{P}\left(V^{\vee}\right)=\mathbb{P}\left(\mathcal{O}_{\mathbb{P}^{1}}(-1)^{2} \oplus \mathcal{O}_{\mathbb{P}^{1}}^{3}\right) \subset \mathbb{P}\left(\mathcal{O}_{\mathbb{P}^{1}}^{7}\right)
$$

Note that

$$
\Gamma\left(\mathcal{O}_{\mathbb{P}\left(V^{\vee}\right)}(a) \otimes \pi^{*} \mathcal{O}_{\mathbb{P}^{1}}(b)\right)=\Gamma\left(\operatorname{Sym}^{a}(V) \otimes \mathcal{O}_{\mathbb{P}^{1}}(b)\right),
$$

which is nonzero if and only if $a+b \geq 0$, e.g., if $a=2$ and $b=-1$. In this case, we have

$$
\operatorname{Sym}^{2}(V) \otimes \mathcal{O}_{\mathbb{P}^{1}}(-1)=\mathcal{O}_{\mathbb{P}^{1}}(1)^{3} \oplus \mathcal{O}_{\mathbb{P}^{1}}^{6} \oplus \mathcal{O}_{\mathbb{P}^{1}}(-1)^{6},
$$

i.e., the space of sections has dimension 12. Furthermore, these all vanish along the distinguished two-dimensional projective subbundle of $\mathbb{P}\left(V^{\vee}\right)$. Since non-singular quadric threefolds contain no two-planes, divisors $D$ in $\mathcal{O}_{\mathbb{P}\left(V^{\vee}\right)(2)} \otimes \pi^{*} \mathcal{O}_{\mathbb{P}^{1}}(-1)$ are fibered over $\mathbb{P}^{1}$ in singular quadric hypersurfaces.

Let $\mathcal{X}=D \cap Q$ where $Q$ has bidegree $(0,2)$, i.e., $Q$ is the pullback of a quadric from $\mathbb{P}^{6}$. The image of $\mathbb{P}\left(V^{\vee}\right)$ in $\mathbb{P}^{6}$ is a quadric hypersurface.

Since $\mathcal{X}$ is contained in a singular quadric hypersurface, its monodromy is non-maximal; indeed, $W\left(D_{5}\right)$ acts on the five singular quadric hypersurfaces containing $\mathcal{X}$ via the canonical homomorphism $W\left(D_{5}\right) \rightarrow$ $\mathfrak{S}_{5}$. This is consistent with Proposition 10, 
QUARTIC DEL PEZZO SURFACES OVER FUNCTION FIELDS OF CURVES 15

Remark 16. Let $C$ denote the intersection of the distinguished twoplane in $\mathbb{P}^{4}$ with $Q$; we have a constant subfamily

$$
C \times \mathbb{P}^{1} \subset \mathcal{X}
$$

It follows that $\mathcal{X} \rightarrow \mathbb{P}^{1}$ admits sections over any ground field.

Case 4. Consider

$$
\mathcal{X} \subset \mathbb{P}^{1} \times \mathbb{P}^{7}
$$

as a complete intersection of three forms $L_{1}, L_{2}$, and $L_{3}$ of bidegree $(1,1)$ and two additional forms quadratic in the second factor. Let $V$ be defined by the exact sequence

$$
0 \rightarrow \mathcal{O}_{\mathbb{P}^{1}}(-1)^{3} \stackrel{\cdot\left(L_{1}, L_{2}, L_{3}\right)}{\rightarrow} \Gamma\left(\mathcal{O}_{\mathbb{P}^{7}}(1)\right) \otimes \mathcal{O}_{\mathbb{P}^{1}} \rightarrow V \rightarrow 0
$$

for generic $L_{i}$, we have

$$
V \simeq \mathcal{O}_{\mathbb{P}^{1}}(1)^{3} \oplus \mathcal{O}_{\mathbb{P}^{1}}^{2}
$$

Even part. Suppose that the additional quadratic forms both have degree $(n, 2)$. Then $\omega_{\pi} \simeq \mathcal{O}_{\mathcal{X}}(2 n+3,-1)$ whence

$$
\pi_{*} \omega_{\pi}^{-1} \simeq \mathcal{O}_{\mathbb{P}^{1}}(-2 n-2)^{3} \oplus \mathcal{O}_{\mathbb{P}^{1}}(-2 n-3)^{2}
$$

and

$$
\operatorname{deg}\left(\pi_{*} \omega_{\pi}^{-1}\right)=-10 n-12, \quad h(\mathcal{X})=20 n+24 .
$$

Example 17 ( $n=-1$ case). Consider the intersection of the forms $L_{1}, L_{2}$, and $L_{3}$ :

$$
\mathbb{P}\left(V^{\vee}\right)=\mathbb{P}\left(\mathcal{O}_{\mathbb{P}^{1}}(-1)^{3} \oplus \mathcal{O}_{\mathbb{P}^{1}}^{2}\right) \subset \mathbb{P}\left(\mathcal{O}_{\mathbb{P}^{1}}^{8}\right) .
$$

In this case, we have

$$
\operatorname{Sym}^{2}(V) \otimes \mathcal{O}_{\mathbb{P}^{1}}(-1)=\mathcal{O}_{\mathbb{P}^{1}}(1)^{6} \oplus \mathcal{O}_{\mathbb{P}^{1}}^{6} \oplus \mathcal{O}_{\mathbb{P}^{1}}(-1)^{3},
$$

i.e., the space of sections has dimension 18. Furthermore, these all vanish along the projectivization of the distinguished one-dimensional projective subbundle of $\mathbb{P}\left(V^{\vee}\right)$. Divisors $D$ in $\mathcal{O}_{\mathbb{P}\left(V^{\vee}\right)(2)} \otimes \pi^{*} \mathcal{O}_{\mathbb{P}^{1}}(-1)$ are fibered over $\mathbb{P}^{1}$ in quadrics containing a distinguished line $\ell$. Let $\mathcal{X}=D_{1} \cap D_{2}$ for two divisors in this linear series.

Remark 18. Since $D_{1}$ and $D_{2}$ both contain $\ell$, we have a constant subfamily:

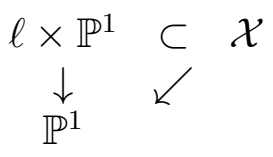

This provides sections for $\mathcal{X} \rightarrow \mathbb{P}^{1}$. Note here that $\mathcal{X}$ is non-minimal, as $\ell$ can be blown down over the ground field. 
Odd part. Suppose that the additional forms have degrees $(n, 2)$ and $(n+1,2)$. Then $\omega_{\pi} \simeq \mathcal{O}_{\mathcal{X}}(2 n+4,-1)$ whence

$$
\pi_{*} \omega_{\pi}^{-1} \simeq \mathcal{O}_{\mathbb{P}^{1}}(-2 n-3)^{3} \oplus \mathcal{O}_{\mathbb{P}^{1}}(-2 n-4)^{2}
$$

and

$$
\operatorname{deg}\left(\pi_{*} \omega_{\pi}^{-1}\right)=-10 n-17, \quad h(\mathcal{X})=20 n+34 .
$$

Example 19 ( $n=-1$ case). Retain the notation of Example 17. Here we let $\mathcal{X}=D \cap Q$ where $Q$ has bidegree $(0,2)$. The image of $\mathbb{P}\left(V^{\vee}\right)$ in $\mathbb{P}^{7}$ lies on three quadrics, and is in fact a cone over a Segre threefold.

The intersection of $\ell$ with $Q$ gives two distinguished sections

$$
\sigma_{1}, \sigma_{2}: \mathbb{P}^{1} \rightarrow \mathcal{X}
$$

which may be conjugate over the ground field. We shall study this case in Section 11.

Case 5. Consider

$$
\mathcal{X} \subset \mathbb{P}^{1} \times \mathbb{P}^{8}
$$

as a complete intersection of four forms $L_{1}, L_{2}, L_{3}$ and $L_{4}$ of bidegree $(1,1)$ and two additional forms quadratic in the second factor. Let $V$ be defined by the exact sequence

$$
0 \rightarrow \mathcal{O}_{\mathbb{P}^{1}}(-1)^{4} \stackrel{\cdot\left(L_{1}, L_{2}, L_{3}, L_{4}\right)}{\rightarrow} \Gamma\left(\mathcal{O}_{\mathbb{P}^{8}}(1)\right) \otimes \mathcal{O}_{\mathbb{P}^{1}} \rightarrow V \rightarrow 0
$$

for generic $L_{i}$, we have

$$
V \simeq \mathcal{O}_{\mathbb{P}^{1}}(1)^{4} \oplus \mathcal{O}_{\mathbb{P}^{1}}
$$

Even part. Suppose that the additional quadratic forms both have degree $(n, 2)$. Then $\omega_{\pi} \simeq \mathcal{O}_{\mathcal{X}}(2 n+4,-1)$ whence

$$
\pi_{*} \omega_{\pi}^{-1} \simeq \mathcal{O}_{\mathbb{P}^{1}}(-2 n-3)^{4} \oplus \mathcal{O}_{\mathbb{P}^{1}}(-2 n-4)
$$

and

$$
\operatorname{deg}\left(\pi_{*} \omega_{\pi}^{-1}\right)=-10 n-16, \quad h(\mathcal{X})=20 n+32 .
$$

Example 20 ( $n=-1$ case). Consider the intersection of the forms $L_{1}, L_{2}, L_{3}$ and $L_{4}$ :

$$
\mathbb{P}\left(V^{\vee}\right)=\mathbb{P}\left(\mathcal{O}_{\mathbb{P}^{1}}(-1)^{4} \oplus \mathcal{O}_{\mathbb{P}^{1}}\right) \subset \mathbb{P}\left(\mathcal{O}_{\mathbb{P}^{1}}^{9}\right) .
$$

In this case, we have

$$
\operatorname{Sym}^{2}(V) \otimes \mathcal{O}_{\mathbb{P}^{1}}(-1)=\mathcal{O}_{\mathbb{P}^{1}}(1)^{10} \oplus \mathcal{O}_{\mathbb{P}^{1}}^{4} \oplus \mathcal{O}_{\mathbb{P}^{1}}(-1),
$$

and the space of sections has dimension 24. These all vanish along a distinguished section $\sigma: \mathbb{P}^{1} \rightarrow \mathbb{P}\left(V^{\vee}\right)$, corresponding the projectivization of the trivial summand of $V^{\vee}$; let $D_{1}$ and $D_{2}$ denote two of the 
QUARTIC DEL PEZZO SURFACES OVER FUNCTION FIELDS OF CURVES 17

divisors in this class. Let $\mathcal{X}=D_{1} \cap D_{2}$, which is a quartic del Pezzo fibration.

Remark 21. The section $\sigma$ factors through $\mathcal{X}$. This canonical section is defined over any field of scalars.

Odd part. Suppose that the additional forms have degrees $(n, 2)$ and $(n+1,2)$. Then $\omega_{\pi} \simeq \mathcal{O}_{\mathcal{X}}(2 n+5,-1)$ whence

$$
\pi_{*} \omega_{\pi}^{-1} \simeq \mathcal{O}_{\mathbb{P}^{1}}(-2 n-4)^{4} \oplus \mathcal{O}_{\mathbb{P}^{1}}(-2 n-5)
$$

and

$$
\operatorname{deg}\left(\pi_{*} \omega_{\pi}^{-1}\right)=-10 n-21, \quad h(\mathcal{X})=20 n+42 .
$$

We can realize the $n=-1$ case via constructions similar to Examples 17, 15, 19, and 20.

Example 22. We do not have examples of height-two quartic del Pezzo fibrations $\mathcal{X} \rightarrow \mathbb{P}^{1}$ with smooth total space and fibers having at worst one ordinary double point.

A direct generalization of the approach in Examples 17, 15, 19, and 20 yields a pencil of singular del Pezzo surfaces. The bundle corresponding to the bidegree $(-2,2)$ forms is

$$
\operatorname{Sym}^{2}(V) \otimes \mathcal{O}_{\mathbb{P}^{1}}(-2)=\mathcal{O}_{\mathbb{P}^{1}}^{10} \oplus \mathcal{O}_{\mathbb{P}^{1}}(-1)^{4} \oplus \mathcal{O}_{\mathbb{P}^{1}}(-2),
$$

parametrizing quadric hypersurfaces singular along the distinguished section of $\mathbb{P}\left(V^{\vee}\right)$. The corresponding divisors $D$ depend on 9 parameters. The bundle corresponding to bidegree $(-1,2)$ forms is

$$
\operatorname{Sym}^{2}(V) \otimes \mathcal{O}_{\mathbb{P}^{1}}(-1)=\mathcal{O}_{\mathbb{P}^{1}}(1)^{10} \oplus \mathcal{O}_{\mathbb{P}^{1}}^{4} \oplus \mathcal{O}_{\mathbb{P}^{1}}(-1),
$$

parametrizing quadric hypersurfaces containing the distinguished section. The associated divisor $D^{\prime}$ depends on $24-1-2=21$ parameters. Altogether, this construction depends on

$$
56+9+21-(3+80)=3
$$

parameters, whereas the expected dimension is two. This reflects the higher cohomology of the bundle $\operatorname{Sym}^{2}(V)(-2)$.

\section{Structure of height TWO FAmilies}

We use the notation of Example 22. Projecting from the distinguished section $p: \mathbb{P}^{1} \rightarrow \mathcal{X}$ gives a rational map

$$
\mathbb{P}\left(\mathcal{O}_{\mathbb{P}^{1}}(-1)^{4} \oplus \mathcal{O}_{\mathbb{P}^{1}}\right)-\rightarrow \mathbb{P}\left(\mathcal{O}_{\mathbb{P}^{1}}(-1)^{4}\right) \simeq \mathbb{P}^{3} \times \mathbb{P}^{1}
$$


The image of $D$ under this mapping is a smooth, trivial quadric surface fibration

$$
\mathcal{Q}:=Q \times \mathbb{P}^{1} \subset \mathbb{P}^{3} \times \mathbb{P}^{1} \stackrel{\varpi}{\rightarrow} \mathbb{P}^{1} .
$$

Let $\mathcal{E}=E \times \mathbb{P}^{1}$ denote the exceptional divisor over $p\left(\mathbb{P}^{1}\right) ; E \subset Q$ is a hyperplane section.

The equation of $D^{\prime}$ takes the form

$$
H\left(u_{1}, u_{2}, u_{3}, u_{4}\right) u_{0}+s F\left(u_{1}, u_{2}, u_{3}, u_{4}\right)+t G\left(u_{1}, u_{2}, u_{3}, u_{4}\right)=0,
$$

where $H$ is linear and $F$ and $G$ are quadratic. Here $s$ and $t$ are homogeneous coordinates on $\mathbb{P}^{1}, u_{1}, \ldots, u_{4}$ are homogeneous coordinates on $\mathbb{P}^{3}$, and $\left[u_{0}, u_{1}, \ldots, u_{4}\right]=[1,0, \ldots, 0]$ is the distinguished section. Thus $\mathcal{X} \rightarrow \mathcal{Q}$ blows up the curve

$$
Z=\{H=s F+t G=0\} \subset \mathcal{E}=E \times \mathbb{P}^{1}=\{H=0\},
$$

a type $(4,1)$ curve in $E \times \mathbb{P}^{1} \simeq \mathbb{P}^{1} \times \mathbb{P}^{1}$. The projection $\varpi_{2}: \mathcal{E} \rightarrow E$ induces $Z \simeq E$. In summary, we have

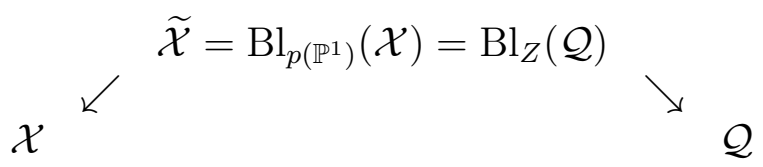

and

$$
h^{1}\left(\Omega_{\widetilde{\mathcal{X}}}^{2}\right)=h^{2}\left(\Omega_{\widetilde{\mathcal{X}}}^{1}\right)=0 .
$$

We enumerate sections $\sigma: \mathbb{P}^{1} \rightarrow \mathcal{X}$ disjoint from the singular section.

height 0: The points of

$$
Q \backslash(Q \cap\{H=0\}) \simeq\left(\mathbb{P}^{1} \times \mathbb{P}^{1}\right) \backslash\{\text { diagonal }\}
$$

yield height-zero sections of $\pi: \mathcal{X} \rightarrow \mathbb{P}^{1}$ disjoint from $p\left(\mathbb{P}^{1}\right)$.

height 1: Fix a line $L \subset Q$ from one of the two rulings, which meets $E$ in a single point. The subfamily $L \times \mathbb{P}^{1} \subset \mathcal{Q}$ meets $Z$ in a single point $z$. Curves of bidegree $(1,1)$ in $L \times \mathbb{P}^{1}$ containing $z$ form a two-parameter family. The proper transforms of these curves in $\mathcal{X}$ are sections disjoint from $p\left(\mathbb{P}^{1}\right)$. Altogether, we obtain a three parameter family of sections, birational to $\mathbb{P}^{1} \times \mathbb{P}^{2}$. Taking the choice of ruling into account, we obtain two such families.

Given a generic point $x^{\prime} \in \mathcal{X}$, we exhibit height-one sections of $\pi$ containing $x^{\prime}$. Regard $x^{\prime} \in Q \times p^{\prime}, p^{\prime}=\pi\left(x^{\prime}\right)$ and choose the ruling so it contains the projection of $x^{\prime}$ onto $Q$. These are indexed by two copies of $\mathbb{P}^{1} \backslash\{$ two points $\}$, one for each ruling of $Q$. 
QUARTIC DEL PEZZO SURFACES OVER FUNCTION FIELDS OF CURVES 19

height 2: Fix a hyperplane section $M \subset Q$, meeting $E$ in two points; $M \times \mathbb{P}^{1}$ meets $Z$ in $\left\{z_{1}, z_{2}\right\}$. Curves of bidegree $(1,1)$ in $M \times \mathbb{P}^{1}$ containing $\left\{z_{1}, z_{2}\right\}$ form a one-parameter family. This construction gives a four parameter family of sections of $\mathcal{X} \rightarrow \mathbb{P}^{1}$, birational to $\mathbb{P}^{3} \times \mathbb{P}^{1}$.

height 3: Repeat the same argument for curves of bidegree $(1,2)$ (twisted cubics) in $Q$.

height 4: Repeat the same argument for curves of bidegree $(1,3)$ in $Q$.

Consider nodal rational curves $M^{\prime} \subset Q$ of bidegree $(2,2)$, which meet $E$ in four points. Let $M$ denote the normalization of $M^{\prime} ; M \times \mathbb{P}^{1}$ meets $Z$ in four points, $\left\{z_{1}, z_{2}, z_{3}, z_{4}\right\}$. Consider curves of bidegree $(1,1)$ in $M \times \mathbb{P}^{1}$ containing these points. Such curves exist only when we have a projective equivalence

$$
\left(M ; z_{1}, \ldots, z_{4}\right) \sim\left(\mathbb{P}^{1}, \varpi\left(z_{1}\right), \varpi\left(z_{2}\right), \varpi\left(z_{3}\right), \varpi\left(z_{4}\right)\right) .
$$

This leads to the following geometric problem:

Let $Q$ be a smooth quadric surface, $E \subset Q$ a smooth hyperplane, $z_{1}, \ldots, z_{4} \in E$ prescribed points, and $w_{1}, \ldots, w_{4} \in$ $\mathbb{P}^{1}$ prescribed points. Describe the morphisms

$$
j: \mathbb{P}^{1} \rightarrow Q
$$

of bidegree $(2,2)$ such that $j\left(w_{i}\right)=z_{i}$ for $i=1, \ldots, 4$.

These are constructed as follows: For any point $q \in Q$, there exists a curve of bidegree $(2,2)$ in $Q$ with a node at $q$, containing $z_{1}, \ldots, z_{4}$, and satisfying the condition on projective equivalence: Indeed, projection from $q$ converts the problem to finding a conic through four given points in $\mathbb{P}^{2}$ so that the points have prescribed projective equivalence class in the conic.

height 5: The case of bidegree $(1,4)$ proceeds just as the case of twisted cubics.

The case of bidegree $(2,3)$ boils down to the following:

Let $Q$ be a smooth quadric surface, $E \subset Q$ a smooth hyperplane, $z_{1}, \ldots, z_{5} \in E$ prescribed points, and $w_{1}, \ldots, w_{5} \in$ $\mathbb{P}^{1}$ prescribed points. Describe the morphisms

$$
j: \mathbb{P}^{1} \rightarrow Q
$$


of bidegree $(2,3)$ such that $j\left(w_{i}\right)=z_{i}$ for $i=1, \ldots, 5$.

These are constructed as follows: Fix pairs of pointed stable curves

$$
\left(E ; z_{1}, \ldots, z_{5}\right), \quad\left(\mathbb{P}^{1} ; w_{1}, \ldots, w_{5}\right), \quad E \simeq \mathbb{P}^{1}
$$

and let $C$ denote the stable curve of genus four obtained by gluing $z_{i}$ to $w_{i}$ for $i=1, \ldots, 5$. The canonical embedding of $C \subset \mathbb{P}^{3}$ is contained in a quadric surface

$$
Q^{\prime} \simeq \mathbb{P}^{1} \times \mathbb{P}^{1} \subset \mathbb{P}^{3}
$$

we may assume the components $E$ and $\mathbb{P}^{1}$ have types $(1,2)$ and $(2,1)$ respectively. For any two general points $e_{1}, e_{2} \in E$, consider the linear series of curves of type $(1,2)$ with assigned basepoints at $e_{1}$ and $e_{2}$. This yields a morphism to a quadric surface

$$
\mathrm{Bl}_{e_{1}, e_{2}} Q^{\prime} \rightarrow Q \subset \mathbb{P}^{3}
$$

The image of $E$ is a hyperplane class; the image of $\mathbb{P}^{1}$ is of type $(2,3)$.

Thus for each collection $\left\{z_{1}, \ldots, z_{5}\right\} \subset E$, we obtain a two-parameter family of sections $\sigma: \mathbb{P}^{1} \rightarrow \mathcal{X}$, corresponding to $M^{\prime} \subset Q$ of bidegree $(2,3)$ such that

- $M^{\prime} \cap Q=\left\{z_{1}, \ldots, z_{5}\right\}$;

- $\left(M ; z_{1}, \ldots, z_{5}\right) \simeq\left(\mathbb{P}^{1} ; \pi\left(z_{1}\right), \ldots, \pi\left(z_{5}\right)\right)$.

The two parameters reflect the choice of $\left(e_{1}, e_{2}\right)$ above. Hence we get a seven-dimensional rational variety parametrizing these sections.

height 6 : We focus on the case of bidegree $(3,3)$, which leads to the following statment:

Let $Q$ be a smooth quadric surface, $E \subset Q$ a smooth hyperplane, $z_{1}, \ldots, z_{6} \in E$ prescribed points, and $\phi$ : $E \simeq \mathbb{P}^{1} \rightarrow \mathbb{P}^{1}$ a fixed mapping of degree four. The morphisms

$$
j: \mathbb{P}^{1} \rightarrow Q
$$

of bidegree $(3,3)$ such that

$$
\left(\mathbb{P}^{1} ; \phi\left(z_{1}\right), \ldots, \phi\left(z_{6}\right)\right) \sim\left(E ; z_{1}, \ldots, z_{6}\right)
$$

are parametrized by a rational variety.

The rational curves of bidegree $(3,3)$ through six fixed points depend on $15-4-6=5$ parameters.

This is proved as follows: Let $C$ be a smooth projective curve of genus five that is Brill-Noether general, i.e., not hyperelliptic, trigonal, 
QUARTIC DEL PEZZO SURFACES OVER FUNCTION FIELDS OF CURVES 21

or a plane quintic. Then the canonical embedding

$$
C \hookrightarrow \mathbb{P}^{4}
$$

realizes $C$ as a complete intersection of three quadrics. In particular, $C$ admits embeddings into a family of quadric del Pezzo surfaces parametrized by $\mathbb{P}\left(I_{C}(2)^{\vee}\right) \simeq \mathbb{P}^{2}$. This remains true for certain stable curves, e.g.,

$$
C=C_{1} \cup_{z_{1}, \ldots, z_{6}} C_{2}, \quad C_{1} \simeq C_{2} \simeq \mathbb{P}^{1},
$$

where $z_{1}, \ldots, z_{6}$ are chosen arbitrarily on each component.

Fixing $\left(C_{1} ; z_{1}, \ldots, z_{6}\right)$ and $\left(C_{2} ; z_{1}, \ldots, z_{6}\right)$, let $S$ denote one of the del Pezzo surfaces arising from this construction. The linear series $\left|C_{1}\right|$ induces a birational morphism onto a quadric

$$
S \rightarrow Q \text {. }
$$

The image of $C_{1}$ is $Q$ is a hyperplane section; the image of $C_{2}$ is a bidegree $(3,3)$ curve with four nodes.

\section{Structure of height Four families}

We give additional details about the geometric structure of height four families. Recall the notation of Example 17. The generic fiber $X$ contains a line $\ell$. Projecting from this line gives a birational morphism

$$
X \rightarrow \mathbb{P}^{2}
$$

blowing down the five lines incident to $\ell ; \ell$ is mapped to the plane conic containing the images of the five lines.

We interpret this for $\mathcal{X}$ over $\mathbb{P}^{1}$ : Fiberwise projection from $\ell \times \mathbb{P}^{1}$ induces a rational map

$$
\mathbb{P}\left(V^{\vee}\right)=\mathbb{P}\left(\mathcal{O}_{\mathbb{P}^{1}}(-1)^{3} \oplus \mathcal{O}_{\mathbb{P}^{1}}^{2}\right) \rightarrow \mathbb{P}\left(\mathcal{O}_{\mathbb{P}^{1}}(-1)^{3}\right) \simeq \mathbb{P}^{2} \times \mathbb{P}^{1}
$$

inducing a birational morphism

$$
\beta: \mathcal{X} \rightarrow \mathbb{P}^{2} \times \mathbb{P}^{1}
$$

The image $L:=\beta\left(\ell \times \mathbb{P}^{1}\right) \subset \mathbb{P}^{2} \times \mathbb{P}^{1}$ is a product $\{$ conic $\} \times \mathbb{P}^{1}$. Let $\varpi_{j}, j=1,2$ denote the projections from $\mathbb{P}^{2} \times \mathbb{P}^{1}$.

We describe the center $Z$ of $\beta$ : It is a rational curve of bidegree $(5,1)$ in $\ell \times \mathbb{P}^{1}$, i.e., projection onto the second factor has degree five and eight branch points. It follows that

$$
h^{2}\left(\Omega_{\mathcal{X}}^{1}\right)=0, \quad h^{1}\left(\Omega_{\mathcal{X}}^{1}\right)=3 .
$$

The composition

$$
\mathbb{P}^{2} \times \mathbb{P}^{1} \stackrel{\beta^{-1}}{-\rightarrow} \mathcal{X} \stackrel{\pi_{2}}{\rightarrow} \mathcal{Y}
$$


is given by forms of bidegree $(3,1)$ on $\mathbb{P}^{2} \times \mathbb{P}^{1}$ vanishing along $Z$. Its restriction to $\ell \times \mathbb{P}^{1} \subset \mathcal{X}$ has bidegree $(2 \cdot 3,1)-(5,1)=(1,1)$, thus collapses $\ell \times \mathbb{P}^{1}$ onto the first factor.

We enumerate sections of $\pi: \mathcal{X} \rightarrow \mathbb{P}^{1}$ of small height $h$, restricting our attention to sections that dominate $\mathcal{X}$. For example, we do not consider sections contained in $\ell \times \mathbb{P}^{1}$. Furthermore, we subdivide based on how the sections meet $\ell \times \mathbb{P}^{1}$ and $\mathcal{E}$, the exceptional divisor of $\beta$.

height 0 : The constant sections of $\mathbb{P}^{2} \times \mathbb{P}^{1} \rightarrow \mathbb{P}^{1}$ induce a twoparameter family of sections for $\pi: \mathcal{X} \rightarrow \mathbb{P}^{1}$, disjoint from $\ell \times \mathbb{P}^{1}$ and $\mathcal{E}$.

height 1: Consider sections of $\varpi_{2}: \mathbb{P}^{2} \times \mathbb{P}^{1} \rightarrow \mathbb{P}^{1}$ projecting onto a line $L \subset \mathbb{P}^{2}$, meeting $Z$ in $\left\{z_{1}, z_{2}\right\}$ with $\varpi_{1}\left(z_{1}, z_{2}\right)=L \cap \ell$. These depend on three parameters, are disjoint from $\ell \times \mathbb{P}^{1}$, but meet $\mathcal{E}$ twice. These sections are parametrized by a space fibered over $\operatorname{Sym}^{2}(Z) \simeq \mathbb{P}^{2}$, with generic fiber $\mathbb{P}^{1}$.

height 2: We have sections of $\varpi_{2}$ projecting onto a line $L$, meeting $Z$ at one point. The resulting sections of $\mathcal{X} \rightarrow \mathbb{P}^{1}$ depend on four parameters, and meet both $\mathcal{E}$ and $\ell \times \mathbb{P}^{1}$ once.

There are also sections with $\pi_{1}\left(\sigma\left(\mathbb{P}^{1}\right)\right)=M$ a plane conic, where the corresponding curve in $M \times \mathbb{P}^{1}$ meets $Z$ in four points $\left\{z_{1}, z_{2}, z_{3}, z_{4}\right\}$ with $\pi_{1}\left(z_{1}, z_{2}, z_{3}, z_{4}\right)=M \times \ell$. These are birational to $\operatorname{Sym}^{4}(Z)$; indeed, there exists a unique plane conic $M \supset \varpi_{1}\left(z_{1}\right), \ldots, \varpi_{1}\left(z_{4}\right)$ such that the projective invariant of these four points in $M$ equals the projective invariant of the points $\pi\left(z_{1}\right), \ldots, \pi\left(z_{4}\right)$ in $\mathbb{P}^{1}$.

height 3: Sections of $\varpi_{2}$ projecting onto a line and disjoint from $Z$. These are parametrized by an open subset $\left(\mathbb{P}^{2}\right)^{\vee} \times \mathbb{P}^{3}$; the generic fiber over a point of $\left(\mathbb{P}^{2}\right)^{\vee}$ is isomorphic to $\mathbb{P}^{3} \backslash\{$ two hyperplanes $\}$. The resulting sections of $\pi$ are disjoint from $\mathcal{E}$ and meet $\ell \times \mathbb{P}^{1}$ twice.

There are sections of $\varpi_{2}$ projecting onto a conic, meeting $Z$ in three points. We have a unique such section over each conic in $\mathbb{P}^{2}$, so the space of these sections is birational to $\mathbb{P}^{5}$. The corresponding sections of $\pi$ meet $\mathcal{E}$ three times and $\ell \times \mathbb{P}^{1}$ one.

Sections of $\varpi_{2}$ projecting onto a nodal cubic curve $M^{\prime}$ with normalization $M$; the corresponding curve in $M \times \mathbb{P}^{1}$ meets $Z$ in six points $\left\{z_{1}, \ldots, z_{6}\right\}$. We have the constraint that

$$
\left(M ; z_{1}, \ldots, z_{6}\right) \sim\left(\mathbb{P}^{1} ; \pi\left(z_{1}\right), \ldots, \pi\left(z_{6}\right)\right),
$$


QUARTIC DEL PEZZO SURFACES OVER FUNCTION FIELDS OF CURVES 23

i.e., these are projectively equivalent. Projection from the node of $M^{\prime}$ induces a degree-two cover

$$
\ell \rightarrow M
$$

and $\ell \simeq Z$ via $\varpi_{1}$. This translates into the following problem

Fix a degree five morphism $\phi: \mathbb{P}^{1} \rightarrow \mathbb{P}^{1}$. Characterize sets of points

$$
\left(z_{1}, \ldots, z_{6}\right) \in\left(\mathbb{P}^{1}\right)^{6}
$$

such that there exists some degree-two morphism $\psi$ :

$\mathbb{P}^{1} \rightarrow \mathbb{P}^{1}$ with

$$
\left(\mathbb{P}^{1} ; \phi\left(z_{1}\right), \ldots, \phi\left(z_{6}\right)\right)=\left(\mathbb{P}^{1} ; \psi\left(z_{1}\right), \ldots, \psi\left(z_{6}\right)\right) .
$$

Note that $\psi$ coincides with projection from the node of $M^{\prime}$ and determines this node uniquely.

This example can be approached using the Brill-Noether analysis employed in Section 5. Consider pairs of 6-pointed stable curves of genus zero

$$
\left(C_{1} ; z_{1}, z_{2}, \ldots, z_{6}\right), \quad\left(C_{2} ; z_{1}, z_{2}, \ldots, z_{6}\right),
$$

and let $C$ denote the genus-five stable curve obtained by gluing these together. If $C$ is immersed in $\mathbb{P}^{2}$ as the union of a conic and a nodal cubic

$$
M^{\prime} \cup \ell \subset \mathbb{P}^{2}
$$

then the canonical curve $j: C \hookrightarrow \mathbb{P}^{4}$ lies on a cubic ruled surface, i.e., the blow up of $\mathbb{P}^{2}$ at the node. This is equivalent to $C$ being trigonal, with the $g_{3}^{1}$ given by the rulings. Trigonal curves are codimension one in the moduli space of curves of genus five.

The projection of the trigonal curves $C$ as above to $M_{0,6} \times M_{0,6}$ is finite onto its image, by a direct computation with MAPLE. This image is unirational as it is dominated by $\mathbb{P}^{5} \times \mathbb{P}^{6}$ — the choice of a plane conic and a plane cubic with a node at a prescribed point.

\section{Structure of height Six families}

Recall the notation leading into Remark 16. In particular, we have

$$
\mathbb{P}\left(V^{\vee}\right)=\mathbb{P}\left(\mathcal{O}_{\mathbb{P}^{1}}(-1)^{2} \oplus \mathcal{O}_{\mathbb{P}^{1}}^{3}\right) \stackrel{\pi_{2}}{\rightarrow} \mathbb{P}^{6},
$$

where the image is quadric hypersurface. It contains a distinguished plane $\Pi=\pi_{2}\left(\mathbb{P}\left(\mathcal{O}_{\mathbb{P}^{1}}^{3}\right)\right)$. The divisor $D \subset \mathbb{P}\left(V^{\vee}\right)$ has image $\pi_{2}(D)$ isomorphic to a cone over the Segre threefold $\mathbb{P}^{1} \times \mathbb{P}^{2} \subset \mathbb{P}^{5}$. The plane $\Pi$ is the cone over $\mathbb{P}^{1} \times\{p\}$ for a point $p \in \mathbb{P}^{2}$. 
Since $\mathcal{Y}$ is the intersection of $\pi_{2}(D)$ with a quadric hypersurface $Q$, we may interpret it as a double cover of $\mathbb{P}^{1} \times \mathbb{P}^{2}$ branched over a divisor of bidegree $(2,2)$. In particular, $\mathcal{Y}$ is a quadric surface bundle over $\mathbb{P}^{1}$ with six degenerate fibers, and a conic bundle over $\mathbb{P}^{2}$, with discriminant a plane quartic $Z \subset \mathbb{P}^{2}$ and associated étale double cover $\gamma: \tilde{Z} \rightarrow Z$. Thus the intermediate Jacobian

$$
\mathrm{IJ}(\mathcal{Y})=\operatorname{Prym}(\tilde{Z} \rightarrow Z)=\mathrm{J}(C),
$$

where $C$ is the genus two curve branched over the discriminant of $\mathcal{Y} \rightarrow$ $\mathbb{P}^{1}$. Thus in particular, we have

$$
h^{2}\left(\Omega_{\mathcal{X}}^{1}\right)=2, h^{1}\left(\Omega_{\mathcal{X}}^{1}\right)=3
$$

which reflects the fact that the fibration $\pi: \mathcal{X} \rightarrow \mathbb{P}^{1}$ has relative Picard rank two.

The morphism $\pi_{2}: \mathcal{X} \rightarrow \mathcal{Y}$ is the blow up of $\mathcal{Y}$ along the curve $T=\Pi \cap Q$, which coincides with the fiber of $\mathcal{Y} \rightarrow \mathbb{P}^{2}$ over $p$. The fibration

$$
\pi=\pi_{1}: \mathcal{X} \rightarrow \mathbb{P}^{1}
$$

is induced by the projection from $p$

$$
\varpi_{p}: \mathbb{P}^{2} \rightarrow \mathbb{P}^{1}
$$

We construct a family of sections of $\pi: \mathcal{X} \rightarrow \mathbb{P}^{1}$ mapping birationally onto $\operatorname{IJ}(\mathcal{X}) \simeq \operatorname{Prym}(\tilde{Z} \rightarrow Z)$. Let $\check{\mathbb{P}}^{2}$ denote the projective space dual to the $\mathbb{P}^{2}$ arising as a factor of the Segre threefold. For each $L \in \check{\mathbb{P}}^{2}$, we have a conic bundle

$$
\phi_{L}: \mathcal{Y} \times_{\mathbb{P}^{2}} L \rightarrow L
$$

degenerate over $L \cap Z$. This conic bundle has eight $(-1)$-curves $E_{1}, \ldots, E_{8}$ that induce sections $\left\{s_{1}, \ldots, s_{8}\right\}$ of $\phi_{L}$; we obtain a generically finite morphism

$$
\mathcal{E} \rightarrow \check{\mathbb{P}}^{2}
$$

where $\mathcal{E}$ is a smooth projective surface compactifying the space parametrizing $E_{1}, \ldots, E_{8}$ as $L$ varies. In particular, there is an open set $\mathcal{E}^{\circ} \subset \mathcal{E}$ and a correspondence

$$
\begin{gathered}
\mathcal{S} \\
\downarrow \\
\mathcal{E}^{\circ}
\end{gathered}
$$

where the vertical arrow is a $\mathbb{P}^{1}$-bundle and the horizontal arrow is induced by the inclusions $E_{1} \cup \ldots \cup E_{8} \subset \mathcal{Y} \times_{\mathbb{P}^{2}} L \subset \mathcal{Y}$. 
QUARTIC DEL PEZZO SURFACES OVER FUNCTION FIELDS OF CURVES 25

Now each $E_{j}$ meets each of the four degenerate fibers of $\phi_{L}$ in one component; these components are indexed by $z_{1}, z_{2}, z_{3}, z_{4} \in \tilde{Z}$. Note that $\gamma\left(z_{1}, z_{2}, z_{3}, z_{4}\right)=L \cap Z \in\left|K_{Z}\right|$. Thus we obtain maps

$$
\mathcal{E} \rightarrow \operatorname{Pic}^{4}(\tilde{Z}) \stackrel{\gamma_{*}}{\rightarrow} \operatorname{Pic}^{4}(Z),
$$

where the image of $\mathcal{E}$ lies in the fiber over $K_{Z}$. Thus there is an induced mapping

$$
\mathcal{E} \rightarrow \operatorname{Prym}(\tilde{Z} \rightarrow Z)
$$

which is clearly birational.

We claim that the fibers of $\mathcal{S} \rightarrow \mathcal{E}$ yield sections of $\pi: \mathcal{X} \rightarrow \mathbb{P}^{1}$, i.e., $\mathcal{E}$ parametrizes a family of sections birational to $\mathrm{IJ}(\mathcal{X})$. Indeed, fix $\left[E_{j}\right] \in \mathcal{E}$ over $L \subset \mathbb{P}^{2}$ and a fiber $\mathcal{X}_{r}=\pi^{-1}(r)$ for $r \in \mathbb{P}^{1}$. The point $r$ corresponds to a line

$$
\ell_{r}:=\varpi_{p}^{-1}(r) \subset \mathbb{P}^{2}
$$

passing through $p$. There is a unique intersection point $p^{\prime}=L \cap \ell_{r}$, and the fiber $\mathcal{Y} \times_{\mathbb{P}^{2}}\left\{p^{\prime}\right\}$ meets $E_{j}$ in precisely one point.

\section{Structure of height eight families}

We return to the example discussed in Remark 14: Let $\mathcal{Y} \subset \mathbb{P}^{5}$ denote a complete intersection of two quadrics, $E \subset \mathcal{Y}$ a smooth codimension-two linear section, and $\mathcal{X}=\mathrm{Bl}_{E}(\mathcal{Y})$. The pencil of linear sections containing $E$ induced a del Pezzo fibration

$$
\pi: \mathcal{X} \rightarrow \mathbb{P}^{1} \text {. }
$$

We review the classical constructions for the lines on $\mathcal{Y}$, following Miles Reid's thesis Rei72] (see also Tju75 and Cas04 for higherdegree curves). The discriminant divisor restricted to the pencil of quadrics yields six points on $\mathbb{P}^{1}$, thus determining a genus two curve $C$. Note that the space of maximal isotropic subspaces on quadrics in the pencil is a $\mathbb{P}^{3}$-bundle over $C$; these correspond to the conics on $\mathcal{Y}$. Projecting from a line $\ell \subset \mathcal{Y}$, we obtain a morphism

$$
\mathrm{Bl}_{\ell}(\mathcal{Y}) \rightarrow \mathbb{P}^{3}
$$

blowing up $C$, realized as a quintic curve in $\mathbb{P}^{3}$. The space of all lines

$$
F_{1}(\mathcal{Y}) \simeq \mathrm{J}(C)
$$

which in turn is isomorphic to the intermediate Jacobian $\operatorname{IJ}(\mathcal{Y})$. Thus we conclude

$$
\mathrm{IJ}(\mathcal{X})=\mathrm{J}(C) \times E .
$$

We examine sections of $\pi$ of low degree. 
(1) lines on $\mathcal{Y}$;

(2) conics on $\mathcal{Y}$ incident to $E$;

(3) twisted cubics on $\mathcal{Y}$ incident to $E$ in two points.

The first family is indexed by $\operatorname{IJ}(\mathcal{Y})$. The second family has dimension three, thus a priori has a chance to dominate $\operatorname{IJ}(\mathcal{X})$. However, the conics in $\mathcal{Y}$ fail to dominate $\operatorname{IJ}(\mathcal{Y})$, so the subfamily we're considering cannot possibly dominate $\operatorname{IJ}(\mathcal{X})$. The family of twisted cubics in $\mathcal{Y}$ is birational to a Grassmann-bundle over the lines in $\mathcal{Y}$ : given $\ell \subset \mathcal{Y}$, each subspace

$$
\ell \subset \mathbb{P}^{3} \subset \mathbb{P}^{5}
$$

intersects $\mathcal{Y}$ in the union of $\ell$ and a twisted cubic $R$. Given $e_{1}, e_{2} \in E$, consider

$$
\operatorname{span}\left(\ell, e_{1}, e_{2}\right) \cap \mathcal{Y}=\ell \cup R,
$$

which necessarily meets $E$ in $e_{1}$ and $e_{2}$. The curves produced in this way are parametrized by

$$
\mathrm{J}(C) \times \operatorname{Sym}^{2}(E),
$$

a $\mathbb{P}^{1}$-bundle over $\mathrm{J}(C) \times E$.

These one-parameter families of sections sweep out a distinguished rational curve in $X$, the generic fiber of $\mathcal{X} \rightarrow \mathbb{P}^{1}$. Fix a pair $(Q, R)$ consisting of

- a quadric surface $E \subset Q \subset \operatorname{span}(Q)$;

- a ruling $R \in \operatorname{Pic}(Q)$ such that $\mathcal{O}_{Q}(R) \mid E=\mathcal{O}_{E}\left(e_{1}+e_{2}\right)$.

There is a natural bijection between such pairs and elements of $\operatorname{Pic}^{2}(E)$. Consider the singular quadric $C_{\ell}(Q) \subset \mathbb{P}^{5}$ that is a cone over $Q$ with vertex $\ell$. The intersection of $C_{\ell}(Q)$ with a fiber $\mathcal{X}_{t}$ of $\mathcal{X} \rightarrow \mathbb{P}^{1}$ consists of $E$ plus a hyperplane section singular at $\ell \cap \mathcal{X}_{t}$.

\section{Structure of height ten families}

Let $Q \subset \mathbb{P}^{4}$ be a smooth quadric threefold. Consider a pencil of quadrics on $Q$ with smooth base locus $C$, a canonical curve of genus 5 . Thus we have a del Pezzo fibration

$$
\pi: \mathcal{X}:=\mathrm{Bl}_{C}(Q) \rightarrow \mathbb{P}^{1}
$$

We assume the fibers have at most one ordinary double point.

Proposition 23. $\pi$ has a family of sections parametrized by a principal homogeneous space over the Jacobian of $C$. 
QUARTIC DEL PEZZO SURFACES OVER FUNCTION FIELDS OF CURVES 27

\begin{tabular}{ccc} 
degree of $R$ & $\#(R \cap C)$ & number of parameters \\
\hline 0 & $\mathrm{NA}$ & 1 \\
1 & 1 & 2 \\
2 & 3 & 3 \\
3 & 5 & 4 \\
4 & 7 & 5 \\
$d$ & $2 d-1$ & $d+1$
\end{tabular}

Figure 2. Parameter counts for spaces of sections

Proof. Figure 2 lists numerical possibilities for the spaces of sections, expressed in terms of the geometry of the associated curve $R \subset Q$.

We focus on the latter case, as there are enough free parameters for the family to dominate $\operatorname{Pic}^{7}(C)$.

Thus we are left with an enumerative problem:

Fix $D \in \operatorname{Pic}^{7}(C)$; then there exists a unique twisted quartic $R \in Q$ such that $R \cap C \in|D|$.

Consider the linear series $|D|$. Through each collection of seven points $\Sigma=\left\{c_{1}, \ldots, c_{7}\right\}$ in linear general position there passes a unique twisted quartic $R(\Sigma)$. We are interested in the locus

$$
Y=\cup_{\Sigma \in|D|} R(\Sigma) \subset \mathbb{P}^{4} .
$$

The linear system $|D|$ realizes $C$ as a plane septic curve with 10 double points $x_{1}, \ldots, x_{10}$. These depend on a total of $36-10-9=17$ parameters, and $x_{1}, \ldots, x_{10}$ can be in general position. Consider the image of $\mathbb{P}^{2}$ under the linear series of quartics through $x_{1}, \ldots, x_{10}$, i.e., the adjoint linear series. The resulting surface $Y$ has degree 6 in $\mathbb{P}^{4}$.

The quadric hypersurface $Q$ pulls back to a plane curve of degree eight double at $x_{1}, \ldots, x_{10}$. Of course, this contains $C$ and the residual curve is a line in $\mathbb{P}^{2}$. Thus we find

$$
Y \cap Q=C \cup R
$$

where $R$ is a twisted quartic in $\mathbb{P}^{4}$.

Now we are essentially done: There exists a family of twisted quartic curves

$$
\begin{array}{r}
\mathcal{R} \\
\downarrow \\
W
\end{array}
$$

whose fibers each meet $C$ seven times. The induced mapping

$$
\mu: W \rightarrow \operatorname{Pic}^{7}(C)
$$


is birational. However, $\operatorname{Pic}^{7}(C)$ is a principal homogeneous space over the Jacobian of $C$.

Remark 24. We summarize the geometric structure of the smalldegree sections listed in Figure 2: When $d=0$ these are just points on $C$, i.e., the constant sections. For $d=1$, we have the lines incident to $C$ contained in $Q$, a $\mathbb{P}^{1}$-bundle over $C$. When $d=2$, we have conics three-secant to $C$, which are birationally parametrized by $\operatorname{Sym}^{3}(C)$; indeed, take the plane spanned by three points on $C$ and intersect this with $Q$.

For $d=3$, we have twisted cubics $R \subset Q$ meeting $C$ in five points, which are birational to a $\mathbb{P}^{1}$-bundle over $\operatorname{Sym}^{3}(C)$. Given such a twisted cubic, let $H \subset \mathbb{P}^{4}$ be the hypersurface it spans; it contains a quadric surface $(Q \cap H)$ and eight distinguished points $(C \cap H)$ on that surface. Five of these points are on $R$; three are not. Thus we have a mapping

$$
\{\text { twisted cubics meeting } C \text { five times }\} \rightarrow-\operatorname{Sym}^{3}(C) \text {. }
$$

The fiber over $p+q+r \in \operatorname{Sym}^{3}(C)$ consists of pairs

$$
\{(H, R): H \supset\{p, q, r\},(C \cap H) \backslash\{p, q, r\} \subset R \subset Q \cap H,
$$

which is a double cover over $\mathbb{P}^{1}$ via projection onto the first factor. Indeed, there are two twisted cubics passing through five generic points on a quadric surface. However, given a pencil of three-planes containing a plane in $\mathbb{P}^{4}$, two elements will be tangent to a quadric hypersurface $Q$. Thus the double cover over $\mathbb{P}^{1}$ is branched in two points, so the fibers of the mapping are rational curves.

Question 25. Where else does this periodic behavior occur for minimal quartic del Pezzo surfaces over $F=k(t)$ ? Can this be analyzed via moduli of vector bundles and Serre's construction?

\section{Structure of FAMilies of Height TWELVE}

Recall the notation of Example 20, We have two projections

$$
\underset{\substack{\downarrow \pi \\ \mathbb{P}^{1}}}{\mathcal{X}} \subset \mathbb{P}\left(V^{\vee}\right)=\mathbb{P}\left(\mathcal{O}_{\mathbb{P}^{1}}(-1)^{4} \oplus \mathcal{O}_{\mathbb{P}^{1}}\right) \stackrel{\pi_{2}}{\rightarrow} \mathbb{P}^{8}
$$

and let $\mathcal{Y}$ denote the image of $\mathcal{X}$ under $\pi_{2}$. The canonical section $\sigma\left(\mathbb{P}^{1}\right)$ is contracted under $\pi_{2}$ to a double point $y \in \mathcal{Y}$. The image is a nodal Fano threefold of genus seven and degree twelve. See [Muk95] for concrete descriptions of smooth Fano varieties of this type. 
QUARTIC DEL PEZZO SURFACES OVER FUNCTION FIELDS OF CURVES 29

There is an alternate approach to $\mathcal{X}$ : Project $\mathcal{X} \rightarrow \mathbb{P}^{1}$ from the distinguished section $\sigma\left(\mathbb{P}^{1}\right)$

$$
\widetilde{\mathcal{X}}=\mathrm{Bl}_{\sigma\left(\mathbb{P}^{1}\right)}(\mathcal{X}) \subset \mathbb{P}\left(\mathcal{O}_{\mathbb{P}^{1}}(-1)^{4}\right) \simeq \mathbb{P}^{1} \times \mathbb{P}^{3} ;
$$

let $\mathcal{E}$ denote the exceptional divisor. The resulting fibration

$$
\tilde{\pi}: \tilde{\mathcal{X}} \rightarrow \mathbb{P}^{1}
$$

is a cubic surface fibration containing a constant line $\mathcal{E}$. Now projection onto the second factor

$$
\tilde{\pi}_{2}: \widetilde{\mathcal{X}} \rightarrow \mathbb{P}^{3}
$$

is birational and collapses $\mathcal{E}$ onto a line $E \subset \mathbb{P}^{3}$. The center of $\tilde{\pi}_{2}$ is the union of $E$ and a curve $C$, of genus seven, admitting a line bundle $M$ with $h^{0}(M, C)=4$, and $\operatorname{deg}(M)=8$, i.e., a $g_{8}^{3}$; the divisor $K_{C}-M$ is a $g_{4}^{1}$. Note that $E$ and $C$ meet in four points $r_{1}, r_{2}, r_{3}$, and $r_{4}$; the fibers of $\tilde{\pi}_{2}$ are mapped onto the pencil of cubic surfaces cutting out $E \cup C$. The birational mapping obtained by composing the inverse of $\tilde{\pi}_{2}$, the blow up of $\mathcal{X}$, and $\pi_{2}$

$$
\mathbb{P}^{3} \rightarrow \mathcal{Y} \subset \mathbb{P}^{8}
$$

is induced by the linear series of quartics vanishing along $C \cup E$.

Note that the intermediate Jacobian $\mathrm{IJ}(\mathcal{X}) \simeq \mathrm{J}(C)$. This also has a Prym interpretation: Projecting from $\mathcal{E}$ fiberwise allows us to realize $\widetilde{\mathcal{X}}$ as a conic bundle

$$
\widetilde{\mathcal{X}} \rightarrow \mathbb{P}^{1} \times \mathbb{P}^{1}
$$

with discriminant a genus-eight curve $D$ of bidegree $(3,5)$. If $\tilde{D} \rightarrow D$ is the cover arising from the conic bundle then

$$
\mathrm{IJ}(\mathcal{X}) \simeq \operatorname{Prym}(\tilde{D} \rightarrow D) .
$$

This is an instance of the tetragonal construction of Donagi and Recillas Don81.

We characterize sections of $\pi$ of small degree, using the geometry sketched above:

(1) the canonical section $\sigma\left(\mathbb{P}^{1}\right)$;

(2) the sections arising from exceptional fibers over points of $C$, which move in a one-parameter family;

(3) the sections arising from secant lines to $C \subset \mathbb{P}^{3}$, which are parametrized by $\operatorname{Sym}^{2}(C)$;

(4) the sections arising from conics five-secant to $C$, which are parametrized by $\operatorname{Sym}^{3}(C)$; 
(5) the sections arising from twisted cubics meeting $C$ in eight points, which are parametrized by $\operatorname{Sym}^{4}(C)$; we explain why this is the case below;

(6) the sections arising from eleven-secant quartic rational curves, which are parametrized by $\operatorname{Sym}^{5}(C)$;

(7) the sections arising from quintic rational curves 14 -secant to $C$, which are parametrized by $\operatorname{Sym}^{6}(C)$;

(8) the sections arising from sextic rational curves 17 -secant to $C$, which are parametrized by $\operatorname{Sym}^{7}(C)$.

First the conic case: each triple of points on $C$ spans a plane, whose residual intersection with $C$ consists of five points that uniquely determine a five-section conic.

We do the cubic case. Given such a section $R$ meeting $C$ in $p_{1}, \ldots, p_{8}$, there is a three-dimensional vector space of quadrics cutting out $R$, which induces a $g_{8}^{2}$ on $C$. Note that the variety of $g_{8}^{2}$ 's on a genus seven curve is four-dimensional, reflecting the fact that a $g_{8}^{2}$ is of the form $K_{C}-c_{1}-c_{2}-c_{3}-c_{4}$ for $c_{1}, c_{2}, c_{3}, c_{4} \in C$. This gives a morphism from the space of sections to $\operatorname{Sym}^{4}(C)$.

Conversely, suppose we are given generic points $c_{1}, c_{2}, c_{3}, c_{4} \in C$. Choose a twisted cubic $R^{\prime}$ incident to $E$ at two points and containing $c_{1}, \ldots, c_{4}$. (The curve $R^{\prime}$ is a tri-section of our original del Pezzo fibration.) The union $R^{\prime} \cup E \cup C$ has degree 12 and arithmetic genus 15, thus

$$
\operatorname{dim} I_{R^{\prime} \cup E \cup C}(4)=35-(48+1-15)=1
$$

and the curve $R^{\prime} \cup E \cup C$ sits on a quartic surface $S$. Note that this generically has Picard lattice:

\begin{tabular}{c|cccc} 
& $h$ & $C$ & $E$ & $R^{\prime}$ \\
\hline$h$ & 4 & 8 & 1 & 3 \\
$C$ & 8 & 12 & 4 & 4 \\
$E$ & 1 & 4 & -2 & 2 \\
$R^{\prime}$ & 3 & 4 & 2 & -2
\end{tabular}

Let $F$ be the residual to $R^{\prime} \cup E$ in a complete intersection of a quadric surface and $S$; this is an elliptic quartic curve. Consider the union $C \cup F \cup E$; we have

$$
\begin{aligned}
h^{0}\left(\mathcal{I}_{C \cup F \cup E}(4)\right) \geq & 35-h^{0}\left(\mathcal{O}_{C \cup F \cup E}(4)\right) \\
& =35-(4 \operatorname{deg}(C \cup F \cup E)+1-\mathrm{g}(C \cup F \cup E)) \\
& =35-(52+1-20)=2 .
\end{aligned}
$$


QUARTIC DEL PEZZO SURFACES OVER FUNCTION FIELDS OF CURVES 31

Let $R$ be residual to $C \cup F \cup E$ in this complete intersection. Note that

$$
C \cdot R=8, \quad R \cdot R=-2,
$$

which gives the desired section.

We turn to the quartic case. First we construct the morphism from the space of sections to $\operatorname{Sym}^{5}(C)$. Suppose that $R$ is a rational quartic curve in $\mathbb{P}^{3}$ meeting $C \subset \mathbb{P}^{3}$ in eleven points. Choose a K3 surface $S$ containing $C$ and $R$, with Picard lattice:

\begin{tabular}{c|ccc} 
& $h$ & $C$ & $R$ \\
\hline$h$ & 4 & 8 & 4 \\
$C$ & 8 & 12 & 11 \\
$R$ & 4 & 11 & -2
\end{tabular}

These exist as

$$
\operatorname{dim} I_{C \cup R}(4) \geq 35-(48+1-17)=3 .
$$

Now $R$ is also contained in a quadric surface $Q$, generally unique. Let $R^{\prime}$ denote the residual to $R$ in $S \cap Q$, so that

$$
h \cdot R^{\prime}=4, \quad R^{\prime} \cdot R^{\prime}=-2, \quad C \cdot R^{\prime}=5 .
$$

The intersection of $C$ with $R^{\prime}$ gives five points on $C$.

We refer the reader to [HT12a, Sect. 8] for the inverse construction.

Now for the case of quintic rational curves: Given six points $c_{1}, \ldots, c_{6} \in$ $C$, let $R^{\prime}$ denote the unique twisted cubic in $\mathbb{P}^{3}$ through these points. Consider the pencil of quartic surfaces containing the union

$$
C \cup_{c_{1}, \ldots, c_{6}} R^{\prime}
$$

each of which has the lattice polarization:

\begin{tabular}{c|ccc} 
& $h$ & $C$ & $R^{\prime}$ \\
\hline$h$ & 4 & 8 & 3 \\
$C$ & 8 & 12 & 6 \\
$R^{\prime}$ & 3 & 6 & -2
\end{tabular}

Consider the residual curve $R$ to $C \cup R^{\prime}$ in this complete intersection; it has genus zero, degree five, and meets $C$ in fourteen points. Thus $R$ corresponds to a section of $\pi$; these are parametrized by elements of $\operatorname{Sym}^{6}(C)$, which may be interpreted as the theta divisor of $\mathrm{J}(C)$. 
Finally, we turn to the case of sextic rational curves. We construct the morphism to $\operatorname{Sym}^{7}(C)$. Suppose that $R$ is 17 -secant to $C$. The union $C \cup R$ has genus 23 and degree 14 so by Riemann-Roch

$$
h^{0}\left(\mathcal{O}_{C \cup R}(4)\right) \geq 56+1-23=34
$$

and thus is contained in a quartic surface $S$. This has Picard group:

\begin{tabular}{c|ccc} 
& $h$ & $C$ & $R$ \\
\hline$h$ & 4 & 8 & 6 \\
$C$ & 8 & 12 & 17 \\
$R$ & 6 & 17 & -2
\end{tabular}

Consider the elliptic fibration $C-h$; it has $R^{\prime}=3 h-R$ as a section

$$
(C-h) \cdot R^{\prime}=1, \quad C \cdot R^{\prime}=7 .
$$

Geometrically, this arises from $R$ via residuation: A rational sextic space curve $R$ satisfies

$$
h^{0}\left(I_{R}(3)\right) \geq 20-(18+1)=1
$$

and thus lies in a cubic surface $T$. Then $R^{\prime}$ is the residual to $R$ in $T \cap S$. The intersection of $R^{\prime}$ with $C$ consists of seven points, which gives us our point in $\operatorname{Sym}^{7}(C)$.

This construction is invertible by the following result, proven in HT12a:

Let $C \subset \mathbb{P}^{3}$ denote a generic tetragonal curve of genus 7 as above, and $c_{1}, \ldots, c_{7} \in C$ generic points. Then there exists a unique sextic rational curve $R^{\prime} \subset \mathbb{P}^{3}$ and quartic K3 surface $S \subset \mathbb{P}^{3}$ such that

$$
R^{\prime} \cap C=\left\{c_{1}, \ldots, c_{7}\right\}
$$

and

$$
C \cup R^{\prime} \subset S \text {. }
$$

Remark 26. There is a straightforward construction of a family of sections parametrized by a rationally connected fibration over $\mathrm{J}(C)$. Fix $c_{1}, \ldots, c_{7} \in C$ and let $R^{\prime \prime}$ denote a rational quartic curve containing these points. Note that the possible such $R^{\prime \prime}$ correspond to ruled quadric surfaces through $c_{1}, \ldots, c_{7}$, a double cover of $\mathbb{P}\left(I_{c_{1}, \ldots, c_{7}}(2)\right)$ branched over a quartic plane curve, i.e., a degree two del Pezzo surface. These curves yield five-sections of $\pi: \mathcal{X} \rightarrow \mathbb{P}^{1}$. Using the argument for Brumer's theorem [Bru78, Lee, for each such odd-degree multisection there exists a family of sections, obtained via a sequence of residuations, and parametrized by a rationally connected variety. 
QUARTIC DEL PEZZO SURFACES OVER FUNCTION FIELDS OF CURVES 33

We summarize the evidence for the existence of sections over generic fibrations of small height collected so far.

\begin{tabular}{lll} 
height & Existence of sections? & Cross reference \\
\hline 0 & always & Remark [12 \\
2 & - & (see Example [22) \\
4 & always & Remark [18 \\
6 & always & Remark [16] \\
8 & always & Remark [14] \\
10 & always & Remark [13 and $₫ 9$ \\
12 & always & Remark [21
\end{tabular}

\section{Fibrations of height Fourteen to tWENty}

Let $\pi: \mathcal{X} \rightarrow \mathbb{P}^{1}$ be a generic quartic del Pezzo fibration of height $14+2 m, m=0,1,2,3$. This corresponds to the following cases of Section 4

- height 14: Example 19] of Case 4;

- height 16: the $n=0$ instance of the even part of Case 3;

- height 18: the $n=0$ instance of the odd part of Case 2;

- height 20: the $n=0$ instance of the odd part of Case 1.

Then we have

$$
\mathcal{X} \subset \mathbb{P}^{1} \times \mathbb{P}^{7-m} \stackrel{\pi_{2}}{\rightarrow} \mathbb{P}^{7-m}
$$

and we write $\mathcal{Y}=\pi_{2}(\mathcal{X})$. We have:

- $\mathcal{Y}$ is anticanonically embedded in $\mathbb{P}^{7-m}$ of degree $10-2 m$;

- $\pi_{2}: \mathcal{X} \rightarrow \mathcal{Y}$ is small, generically contracting $2^{m+1}$ sections of $\pi$ to nodes of $\mathcal{Y}$.

Indeed, the anticanonical divisor of $\mathcal{X}$ (as computed via adjunction) coincides with $\pi_{2}^{*} \mathcal{O}_{\mathbb{P}^{7-m}}(1)$. A direct computation with Bezout's theorem shows that the number of sections contracted by $\pi_{2}$ is as claimed. (Over non-closed fields, there is no reason for these to be defined over the ground field.)

In the following sections, we make the geometry even more explicit.

Height fourteen. Recall the notation from Example 19. The two distinguished sections are denoted $\sigma_{1}, \sigma_{2}: \mathbb{P}^{1} \rightarrow \mathcal{X}$. Projection onto 
the second factor gives

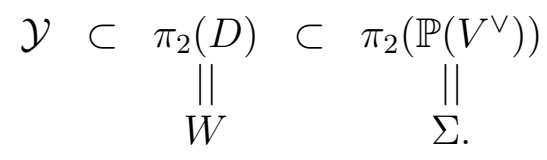

Note that $\Sigma$ is a cone over a Segre threefold $\mathbb{P}^{1} \times \mathbb{P}^{2} \subset \mathbb{P}^{5}$, with vertex $\ell$, a line. The equations of $\Sigma$ are given by the $2 \times 2$ minors of the matrix:

$$
\left(\begin{array}{lll}
u & v & w \\
x & y & z
\end{array}\right) ;
$$

we express

$$
\ell=\{u=v=w=x=y=z=0\} \subset \Sigma .
$$

We may interpret $W$ as follows: Fix a fiber $\{$ pt. $\} \times \mathbb{P}^{2}$ in the Segre threefold, yielding a subspace

$$
\Lambda \simeq \mathbb{P}^{4} \subset \Sigma, \quad \Lambda \supset \ell
$$

Write

$$
\Lambda=\{u=v=w=0\} \subset \Sigma
$$

and

$Q^{\prime}=\left\{q_{1}(u, v, w)+u l_{1}(x, y, z, s, t)+v l_{2}(x, y, z, s, t)+w l_{3}(x, y, z, s, t)=0\right\}$,

where $q_{1}$ is quadratic and the $l_{i}$ are linear. Note that $Q^{\prime}$ is necessarily singular along some line $\ell^{\prime} \subset \Lambda$, and thus has rank at most six; in suitable coordinates, we have

$$
Q^{\prime}=\left\{u m_{1}+v m_{2}+w m_{3}=0\right\},
$$

where the $m_{i}$ are linear. Let $W$ denote the residual to $\Lambda$ in $Q^{\prime} \cap \Sigma$, which is contained in the additional quadric hypersurface

$$
Q^{\prime \prime}=\left\{x m_{1}+y m_{2}+z m_{3}=0\right\} .
$$

Suppose that $\Lambda^{\prime} \simeq \mathbb{P}^{4}$ is a cone over a generic fiber $\{$ pt.' $\} \times \mathbb{P}^{2}$ in the Segre threefold; then $Q^{\prime}$ and $Q^{\prime \prime}$ cut out the same quadric hypersurface on $\Lambda^{\prime}$, i.e.,

$$
Q^{\prime} \cap \Lambda^{\prime}=Q^{\prime \prime} \cap \Lambda^{\prime}
$$

We claim that $W$ is a linear section of the Grassmannian $\operatorname{Gr}(2,5) \subset$ $\mathbb{P}^{9}$. Indeed, let $\xi_{i j}, 1 \leq i<j \leq 5$ denote the Plücker coordinates on the Grassmannian and consider the linear section $\xi_{14}=0$. The Plücker relations specialize to the $2 \times 2$ minors of

$$
\left(\begin{array}{ccc}
\xi_{12} & \xi_{13} & -\xi_{15} \\
\xi_{24} & \xi_{34} & \xi_{45}
\end{array}\right)
$$


QUARTIC DEL PEZZO SURFACES OVER FUNCTION FIELDS OF CURVES 35

as well as

$$
\xi_{12} \xi_{35}-\xi_{13} \xi_{25}+\xi_{15} \xi_{23}=0-\xi_{23} \xi_{45}+\xi_{24} \xi_{35}-\xi_{25} \xi_{34}=0 .
$$

Setting

$$
u=\xi_{12}, v=\xi_{13}, w=-\xi_{15}, x=\xi_{24}, y=\xi_{34}, z=\xi_{45}
$$

and $m_{1}=\xi_{35}, m_{2}=-\xi_{25}$, and $m_{3}=-\xi_{23}$, we obtain the equations for $W$ given above.

In particular, $W$ has degree five. Furthermore, a direct calculation shows that $W$ has ordinary double points along $\ell$. This reflects the fact that the birational morphism $\pi_{2}: D \rightarrow W$ collapses $\ell \times \mathbb{P}^{1}$ to $\ell$, and any small contraction of a smooth variety is singular.

The variety $\mathcal{Y}$ is the intersection of $W$ with a generic quadric $Q$, and thus has degree ten and two ordinary singularities

$$
\left\{y_{1}, y_{2}\right\}=\ell \cap Q=\left\{\pi_{2}\left(\sigma_{1}\left(\mathbb{P}^{1}\right)\right), \pi_{2}\left(\sigma_{2}\left(\mathbb{P}^{1}\right)\right)\right\} .
$$

The dualizing sheaf $\omega_{\mathcal{Y}}=\mathcal{O}_{\mathcal{Y}}(-1)$, so $\mathcal{Y}$ is a Fano variety with ordinary double points, and thus a degeneration of a smooth Fano variety of degree ten and genus six [Nam97]. Degenerations with one node are studied in [DIM11.

We describe the quartic del Pezzo fibration in terms of the geometry of $\mathcal{Y} \subset \mathbb{P}^{7}$. The fibers are obtained by intersecting the subspaces $\Lambda^{\prime}$ specified above (associated with the fibers of $\mathbb{P}^{1} \times \mathbb{P}^{2} \rightarrow \mathbb{P}^{1}$ ) with $Q$ and $Q^{\prime}$; note that $Q^{\prime} \equiv Q^{\prime \prime}\left(\bmod \Lambda^{\prime}\right)$.

Height sixteen. In this case $\mathcal{Y} \subset \mathbb{P}^{6}$ is a complete intersection of three quadrics with four nodes. These have expected dimension

$$
\operatorname{dim} \operatorname{Gr}\left(3, \Gamma\left(\mathcal{O}_{\mathbb{P}^{6}}(2)\right)\right)-\operatorname{dim} \mathrm{PGL}_{7}-4=23,
$$

which is equal to the expected dimension of the space of quartic del Pezzo fibrations of height sixteen.

Height eighteen. Here $\mathcal{Y} \subset \mathbb{P}^{5}$ is a complete intersection of a quadric and cubic with eight nodes. These have expected dimension 26, which is equal to the expected dimension of the space of quartic del Pezzo fibrations of height eighteen.

Height twenty. Here $\mathcal{Y} \subset \mathbb{P}^{4}$ is a quartic threefold with sixteen nodes; however, these have expected dimension 30, greater than the expected dimension $\left(\frac{3}{2} h(\mathcal{X})-1=29\right)$ of the quartic del Pezzo fibrations of height twenty. 
Consider the Grassmannian $\operatorname{Gr}\left(2, \Gamma\left(\mathcal{O}_{\mathbb{P}^{4}}(2)\right)\right)$ parametrizing quartic del Pezzo surfaces. A generic conic curve in this Grassmannian corresponds to the rulings of a nonsingular quadric surface in $\mathbb{P}\left(\Gamma\left(\mathcal{O}_{\mathbb{P}^{4}}(2)\right)\right)$. These can be expressed in equations as

$$
P_{1} s+Q_{1} t=P_{2} s+Q_{2} t=0
$$

where $P_{1}, Q_{1}, P_{2}, Q_{2} \in \Gamma\left(\mathcal{O}_{\mathbb{P}^{4}}(2)\right)$. In other words

$$
\mathcal{X} \subset \mathbb{P}^{1} \times \mathbb{P}^{4}
$$

as a complete intersection of two hypersurfaces of bidegree $(1,2)$. These depend on

$$
2(2 \times 15-2)-(3+24)=29
$$

parameters. Let $\sigma_{1}, \ldots, \sigma_{16}: \mathbb{P}^{1} \rightarrow \mathcal{X}$ denote the constant sections, corresponding to the solutions

$$
\left\{y_{1}, \ldots, y_{16}\right\}=\left\{P_{1}=Q_{1}=P_{2}=Q_{2}=0\right\} .
$$

Projection onto the second factor gives the quartic hypersurface

$$
\mathcal{Y}=\left\{P_{1} Q_{2}-Q_{1} P_{2}=0\right\} \subset \mathbb{P}^{4},
$$

with nodes at $y_{1}, \ldots, y_{16}$.

There is a second del Pezzo fibration

$$
\mathcal{Y} \rightarrow \mathbb{P}^{1}
$$

constructed as follows. Fix a fiber $\mathcal{X}_{t}=\pi^{-1}(t)$ and its image $\mathcal{X}_{t} \subset \mathcal{Y}$. The linear series of quadrics vanishing at $\mathcal{X}_{t}$ gives a mapping $\pi^{\prime}$, whose fibers are also del Pezzo surfaces of degree four. This is a morphism where $\mathcal{X}_{t} \subset \mathcal{Y}$ is Cartier, i.e., away from $y_{1}, \ldots, y_{16}$. There exists a small resolution $\mathcal{X}^{\prime} \rightarrow \mathcal{Y}$ such that the induced

$$
\pi^{\prime}: \mathcal{X}^{\prime} \rightarrow \mathbb{P}^{1}
$$

is a morphism. We obtain $\mathcal{X}^{\prime}$ from $\mathcal{X}$ by flopping each of $\sigma_{1}, \ldots, \sigma_{16}$. Finally, let $\tilde{Y}$ denote the blow up of $\mathcal{Y}$ at each of the $y_{i}$, which coincides with the closure of the graph of the birational mapping $\mathcal{X} \rightarrow-\mathcal{X}^{\prime}$; the induced morphism

$$
\left(\pi, \pi^{\prime}\right): \tilde{Y} \rightarrow \mathbb{P}^{1} \times \mathbb{P}^{1}
$$

has genus five canonical curves as fibers.

Projecting from one of the $\sigma_{j}$, we obtain a conic bundle

$$
\mathcal{W} \rightarrow \mathbb{P}^{1} \times \mathbb{P}^{1}
$$

branched along a curve $D$ of bidegree $(5,5)$, of genus 16 . The Prym variety therefore has dimension 15; see also [Che06], where the nonrationality of a generic $\mathcal{X}$ is established. 
QUARTIC DEL PEZZO SURFACES OVER FUNCTION FIELDS OF CURVES 37

\section{REFERENCES}

[Bak08] H. F. Baker. On the Invariants of a Binary Quintic and the Reality of its Roots. Proc. London Math. Soc., S2-6(1):122, 1908.

[Bea77] Arnaud Beauville. Variétés de Prym et jacobiennes intermédiaires. Ann. Sci. École Norm. Sup. (4), 10(3):309-391, 1977.

[Bru78] Armand Brumer. Remarques sur les couples de formes quadratiques. $C$. R. Acad. Sci. Paris Sér. A-B, 286(16):A679-A681, 1978.

[Cas04] Ana-Maria Castravet. Rational families of vector bundles on curves. Internat. J. Math., 15(1):13-45, 2004.

[Che06] Ivan Cheltsov. Nonrational nodal quartic threefolds. Pacific J. Math., 226(1):65-81, 2006.

[DI87] Pierre Deligne and Luc Illusie. Relèvements modulo $p^{2}$ et décomposition du complexe de de Rham. Invent. Math., 89(2):247-270, 1987.

[DIM11] Olivier Debarre, Atanas Iliev, and Laurent Manivel. On nodal prime Fano threefolds of degree 10. Sci. China Math., 54(8):1591-1609, 2011.

[DIM12] Olivier Debarre, Atanas Iliev, and Laurent Manivel. On the period map for prime Fano threefolds of degree 10. J. Algebraic Geom., 21(1):21-59, 2012.

[dJS03] A. J. de Jong and J. Starr. Every rationally connected variety over the function field of a curve has a rational point. Amer. J. Math., 125(3):567$580,2003$.

[Don81] Ron Donagi. The tetragonal construction. Bull. Amer. Math. Soc. (N.S.), 4(2):181-185, 1981.

[Esn03] Hélène Esnault. Varieties over a finite field with trivial Chow group of 0-cycles have a rational point. Invent. Math., 151(1):187-191, 2003.

[GHS03] Tom Graber, Joe Harris, and Jason Starr. Families of rationally connected varieties. J. Amer. Math. Soc., 16(1):57-67 (electronic), 2003.

[Has09] Brendan Hassett. Rational surfaces over nonclosed fields. In Arithmetic geometry, volume 8 of Clay Math. Proc., pages 155-209. Amer. Math. Soc., Providence, RI, 2009.

[HH] Brendan Hassett and Donghoon Hyeon. Log minimal model program for the moduli space of stable curves: The first flip. To appear in Annals of Mathematics.

[HRS05] Joe Harris, Mike Roth, and Jason Starr. Curves of small degree on cubic threefolds. Rocky Mountain J. Math., 35(3):761-817, 2005.

[HT12a] Brendan Hassett and Yuri Tschinkel. Embedding pointed curves in $K 3$ surfaces, 2012.

[HT12b] Brendan Hassett and Yuri Tschinkel. Spaces of sections of quadric surface fibrations over curves. In Compact moduli spaces and vector bundles, volume 564 of Contemp. Math., pages 227-249. Amer. Math. Soc., Providence, RI, 2012.

[IM00] A. Iliev and D. Markushevich. The Abel-Jacobi map for a cubic threefold and periods of Fano threefolds of degree 14. Doc. Math., 5:23-47 (electronic), 2000. 
[IM07] Atanas Iliev and Dimitri Markushevich. Parametrization of sing $\Theta$ for a Fano 3-fold of genus 7 by moduli of vector bundles. Asian J. Math., 11(3):427-458, 2007.

[JHS] M. Roth J. Harris and J. Starr. Abel-Jacobi maps associated to smooth cubic threefolds. arXiv:math/0202080.

[Kan89] Vassil Kanev. Intermediate Jacobians and Chow groups of three-folds with a pencil of del Pezzo surfaces. Ann. Mat. Pura Appl. (4), 154:13-48, 1989.

[Kir85] Frances Clare Kirwan. Partial desingularisations of quotients of nonsingular varieties and their Betti numbers. Ann. of Math. (2), 122(1):41-85, 1985.

[Kol96] János Kollár. Rational curves on algebraic varieties, volume 32. SpringerVerlag, Berlin, 1996.

[Lan55] Serge Lang. Abelian varieties over finite fields. Proc. Nat. Acad. Sci. U.S.A., 41:174-176, 1955.

[Lee] David B. Leep. The Amer-Brumer theorem over arbitrary fields. Preprint.

[MM93] Toshiki Mabuchi and Shigeru Mukai. Stability and Einstein-Kähler metric of a quartic del Pezzo surface. In Einstein metrics and Yang-Mills connections (Sanda, 1990), volume 145 of Lecture Notes in Pure and Appl. Math., pages 133-160. Dekker, New York, 1993.

[MT01] D. Markushevich and A. S. Tikhomirov. The Abel-Jacobi map of a moduli component of vector bundles on the cubic threefold. J. Algebraic Geom., 10(1):37-62, 2001.

[Muk95] Shigeru Mukai. Curves and symmetric spaces. I. Amer. J. Math., 117(6):1627-1644, 1995.

[Nam97] Yoshinori Namikawa. Smoothing Fano 3-folds. J. Algebraic Geom., 6(2):307-324, 1997.

[Rei72] Miles Reid. The complete intersection of two or more quadrics. PhD thesis, Cambridge University, 1972.

[Sha78] Stephen S. Shatz. On subbundles of vector bundles over $\mathbf{P}^{1}$. J. Pure Appl. Algebra, 10(3):315-322, 1977/78.

[Shr06] K. A. Shramov. On the rationality of nonsingular threefolds with a pencil of del Pezzo surfaces of degree 4. Mat. Sb., 197(1):133-144, 2006.

[Tju75] A. N. Tjurin. The intersection of quadrics. Uspehi Mat. Nauk, 30(6(186)):51-99, 1975.

[Zhu11] Yi Zhu. Homogeneous fibrations over curves, 2011. arXiv:1111.2963v2.

Department of Mathematics, Rice University, MS 136, Houston, TX 77251-1892, USA

E-mail address: hassett@rice.edu

Courant Institute, New York University, New York, NY 10012, USA

E-mail address: tschinkel@cims.nyu.edu

Simons Foundation, 160 Fifth Avenue, New York, NY 10010, USA 